1 Title: Characterization of novel regulatory modules controlling leaf

2 angle in maize

3 Short title: Novel regulatory modules control leaf angle in maize

4 Corresponding authors:

5 Pinghua Li

6 State Key Laboratory of Crop Biology, College of Agronomic Sciences,

7 Shandong Agricultural University, Tai'an, Shandong 271018, China

8 Telephone: +86-538-824-2995

9 Email: pinghuali@sdau.edu.cn

10 Xianglan Wang

11 State Key Laboratory of Crop Biology, College of Agronomic Sciences,

12 Shandong Agricultural University, Tai'an, Shandong 271018, China

13 Telephone: +86-538-824-2995

14 Email: wangxianglan@sdau.edu.cn 


\section{Characterization of novel regulatory modules controlling leaf angle in maize}

Xianglan Wang ${ }^{1 * \#}$, Xiaokun Wang ${ }^{1 *}$, Shilei Sun ${ }^{1}$, Xiaoyu Tu ${ }^{2,3}$, Kande Lin ${ }^{2,3}$, Lei Qin ${ }^{1}$, Xingyun Wang ${ }^{1}$, Gang $\mathrm{Li}^{1}$, Silin Zhong $^{2}$, Pinghua $\mathrm{Li}^{1}{ }^{1 \#}$

${ }^{1}$ State Key Laboratory of Crop Biology, College of Agronomic Sciences, Shandong Agricultural University, Tai'an, Shandong 271018, China

2 State Key Laboratory of Agrobiotechnology, School of Life Sciences, The Chinese University of Hong Kong, Hong Kong, China

${ }^{3}$ The South China Botanical Garden, Chinese Academy of Sciences, Guangzhou, China

* These authors contributed equally to this work.

\#To whom correspondence should be address: Pinghua Li (pinghuali@sdau.edu.cn) or Xianglan Wang (wangxianglan@sdau.edu.cn)

\section{One sentence summary}

The maize leaf angle is regulated by the ZmLG2-ZmBEH1-ZmSCL28 and ZmLG1-ZmBZR1-ZmSCL28 modules.

\section{Footnotes}

\section{List of author contributions}

P.L. and X.W. designed the research; X.W., X.W. S.S., Z.T., K.L., L. Q, X.W. performed the experiments; X.W, X.W., G.L., S. Z. and P. L wrote the article.

\section{Responsibilities of the Author for Contact}

The author responsible for distribution of materials integral to the findings presented in this article in accordance with the policy described in the Instructions for Authors (www.plantphysiol.org) is: Pinghua Li (pinghuali@sdau.edu.cn).

\section{Competing financial interests}

The authors declare no competing financial interests.

\section{Funding}

This work was supported by grants from the National Natural Science Foundation of China (31901552 and 92035302), Hong Kong GRF 14109420 and Hong Kong AoE/M403/16.

\section{Email address of Author for Contact}

Pinghua Li

Email: pinghuali@sdau.edu.cn 


\section{Abstract}

Leaf angle is an important agronomic trait determining maize planting density and light penetration into the canopy, and contributes significantly to the yield gain in modern maize hybrids. However, little is known about its molecular mechanism beyond the Liguless1 (LG1) and Liguless2 (LG2) genes. In this study, we found that transcription factor $\mathrm{ZmBEHI}$ is targeted by ZmLG2 and regulates the leaf angle formation by influencing the sclerenchyma cells layers on the adaxial side. ZmBEH1 can interact with transcription factor ZmBZR1, whose expression is directly activated by ZmLG1. Both ZmBEH1 and ZmBZR1 bind to the promoter of ZmSCL28, the third transcription factors that influences the leaf angle. Our study demonstrates novel regulatory modules controlling leaf angle, and provides new gene editing targets for creating optimal maize architecture suitable for dense-planting.

\section{Introduction}

Maize yields have increased dramatically since the late 1930's due in large part to the increased planting densities (Duvick, 2005). The selection of upright leaf architecture enables plants to be grown at higher density while minimizing the shading of neighboring plants and increasing the efficiency of light capture. Leaf angle, the trait that defined as the inclination between the leaf blade midrib and the vertical culm, contribute significantly to the upright leaf architecture. Recently, the identification of two quantitative trait loci, UPA1 and UPA2, which conferred the upright plant architecture by reducing the leaf angle, and then dramatically improved planting density and enhanced grain yield (Tian et al., 2019), further support the critical role of leaf angle alteration in maize breeding.

The establish of the leaf angle in maize is determined by the ligular region, 
which encompasses the ligule and auricle, serves as a hinge at the bladesheath boundary, allowing the leaf blade to project away from the stem. Two classical genes, Liguless1 (LG1), a Squamosa Promoter Binding Protein domain containing transcription factor (TF) (Moreno et al., 1997) and Liguless2 (LG2), a basic leucine zipper transcription factor family member (Harper and Freeling, 1996; Walsh et al., 1998), play key roles in the ligular region formation that altered the leaf angle. In $\lg 1$ mutant, ligule and auricle are not formed and the plants exhibit excessively erect leaves (Moreno et al., 1997). In $\lg 2$, ligule and auricle are often absent or positioned incorrectly, also cause the upright plant architecture with extreme small leaf angle (Lambert and Johnson, 1978; Walsh et al., 1998; Mantilla-Perez and Salas Fernandez, 2017). Introgression of $\lg 2$ mutant alleles into maize hybrid lines presented an increased grain yield (Pendleton et al., 1968; Lambert and Johnson, 1978), however, the extremely erect leaf limits the commercial use of this allele. Even though, ZmLG1 and ZmLG2 could provide genetic clue to optimize leaf angle for dense planting, such as identify their downstream targets. The genetic basis by which genes affect leaf angle in maize need further exploration.

Several genes in maize have been identified to control leaf angle ( Muehlbauer et al., 1999; Ku et al., 2011; Zhang et al., 2014; Tian et al., 2019; Cao et al., 2020; Ren et al., 2020), and most of them are related to the phytohormone brassinosteroids (BRs), e.g., BRD1 (brassinosteroid C-6 oxidase1) and BRI1 (Brassinosteroid Insensitive 1). The overexpression of $B R D 1$, which encodes brassinosteroid C-6 oxidase1 to catalyze the last step of brassinosteroid synthesis, increased the leaf angle by enlarging auricle and decreasing the number of sclerenchyma cells on the adaxial side; and knockout the function of BRII, which encodes a Leu-rich repeat receptor kinase that responsible for BR binding to start BR signal transduction, exhibit the upright leaf with decreased auricle formation (Makarevitch et al., 2012; Tian et al., 2019). 
The BR singling is send from BRI1 receptor kinase at the cell surface to the BZR1/BES1 (Brassinazole Resistant 1/ BRI1-EMS-Suppressor1) transcription factors, like BZR1 in Arabidopsis, which directly binds to the $\mathrm{BR}$ response element (BRRE, CGTGT/CG) to regulate the expression of the downstream BR-responsive genes ( $\mathrm{Li}$ and Jin, 2007). The BZR1 family members act not only as major transcription factors in BR singling pathway, but also as mediators to participate in plant development and abiotic stress (Wang et al., 2014). In Arabidopsis, BZR1 promotes phloem and xylem Differentiation (Saito et al., 2018), and interacts with GATA2, a transcription factor in the light signaling pathway, to regulate hypocotyl elongation of seedlings (Luo et al., 2010). A recent study showed that anther locule development was also regulated by BZR1 (Chen et al., 2019). In addition, BZR1 serve as regulator in abiotic stress. For instance, BZR1 positively modulates plant freezing tolerance through CBF-dependent and CBFindependent pathways (Li et al., 2017). In rice, OsBZR1 was identified by its homology to Arabidopsis BZR1, and the Loss-of-function of OsBZR1 reduced BR sensitivity, presented dwarfism phenotype with erect leaves, indicating the critical roles of $O s B Z R 1$ in BR signal pathway (Bai et al., 2007). To date, very few researches focus on maize BZR transcription family and our understanding of the role of BZRs in maize is still limited.

In this study, we screened the downstream target of ZmLG2 and identified a BZR1/BES1 homolog gene named ZmBEH1. The loss-of-function of $\mathrm{ZmBEH1}$ exhibits upright plant architecture. Interestingly, the maize ZmBZR1, which also influences the leaf angle, acts downstream of ZmLG1, and interacts with $\mathrm{ZmBEH} 1$ to co-regulate the expression of $\mathrm{ZmSCL28}$, a rice DLT (Tong et al., 2009) homolog that altering the leaf angle in maize. These findings reveal a ZmLLG2/LG1-ZmBEH1/BZR1-ZmSCL28 cascades primarily regulates the leaf angle in maize, which will benefit to deepen our understanding on BR response in maize and help to optimize maize plant architecture for dense planting. 


\section{Results}

\section{ZmLG2 binds to the promoter of $Z m B E H 1$}

ZmLG2, which encodes a bZIP transcription factor, is a classical regulator controlling maize ligule development (Harper and Freeling, 1996; Walsh et al., 1998; Bolduc et al., 2012).

To study the regulatory mechanism mediated by ZmLG2, we searched the maize 104 TF ChIP-Seq data collection (Tu et al., 2020), and found a putative target gene named ZmBEH1 (BZR1/BES1 homolog), which is a homolog to the rice BZR3 (Supplemental Fig. S1), has a strong binding peak of ZmLG2 in front of TSS (Fig. 1A).

To verify the affinity between ZmLG2 and ZmBEH1 promoter, electrophoretic mobility shift assay (EMSA) was conducted using purified recombinant ZmLG2-GST fusion protein and a synthesized 36 bp probe deriving from the promoter of $\mathrm{ZmBEH} 1$ (Material and Methods). As shown in Figure 1B, ZmLG2-GST fusion protein has high affinity with the ZmBEH1 promoter probe, however, this binding was significantly inhibited by unlabeled DNA in the competitive assay, indicating the binding of ZmLG2 to $\mathrm{ZmBEH1} 36 \mathrm{bp}$ promoter probe is highly specific (Fig. 1B). In addition, Dual-luciferase (LUC) transient transcriptional activity assay performed in the maize protoplasts with $\mathrm{ZmLG} 2$ driven by the $35 \mathrm{~S}$ promoter as an effecter and luciferase driven by the $Z m B E H 1$ promoter as the reporter. The result also confirmed that $\mathrm{ZmBEH} 1$ promoter could be activated by $\mathrm{ZmLG} 2$ in vivo (Fig. 1C).

Next, we checked the expression profiles of $\mathrm{ZmBEH1}$ in $\lg 2$ mutant with RNA-seq using ligule tissues from five different maturation stages (from ligule band initiation in S0 to auricle matured in S4, Material and methods, Supplemental Fig. S2, A and C, unpublished). The expression of ZmBEH1 was significantly reduced in $\lg 2$ relative to the wild type (WT) (Fig. 1D). All these results indicate that the $Z m L G 2$ positively regulate the expression $Z m B E H 1$, and $Z m B E H 1$ is the direct downstream target of ZmLG2. 
ZmBEH1 Mu-insertion mutant and CRISPR lines showed reduced leaf angle

To study the function of $\mathrm{ZmBEH} 1$, we obtained the uniform $\mathrm{Mu}$ insertion mutant of ZmBEH1 (named beh1-1) from maize stock center. Genomic DNA and real-time PCR results showed that the $\mathrm{Mu}$ transposon was inserted to the 5'-UTR of ZmBEH1 and dramatically decreased its expression in the mutant (Fig. 2, A and B, Supplemental Fig. S3). The decreased expression of $\mathrm{ZmBEH} 1$ is consistent with the slow growing phenotype of the beh1-1 plants, with the total heights much shorter than that of the WT in both 15-day-old seedlings stage (V2 stage) and 54-day-old stage (V10 stage) (Fig. 2C). Interestingly, when the WT height stopped to increase after silking, beh1-1 mutant could catch up. Another phenotype of beh1-1 was its erect leaves. The leaf angle of the mutants decreased $34.9 \%$ in V2 stage and 30.9\% in V10 stage compared with the WT (Fig. 2D). We noticed a smaller ligular area and margin width in the behl-1 than those in the WT, which decreased the auricle size at V2 and V10 stage and then decreased the leaf angle (Fig. 2E). Crosssections of the ligular region revealed a reduced number of sclerenchyma cell layers at the adaxial blade-sheath junction in the mutant at the midrib (Fig. $2 \mathrm{~F})$, suggesting that in maize, leaf angle is mediated at least in part through the regulation of adaxial sclerenchyma development.

To confirm the function of $Z m B E H 1$, we used CRISPR/Cas9 system to edit ZmBEH1 specifically. The two obtained CRISPR mutants were named beh12 and beh1-3, which have a $76 \mathrm{bp}$ deletion and one base pair change, respectively (Supplemental Fig. S4A). Consistent with the phenotype of beh1-1, beh1-2 and beh1-3 exhibit erect leaves, fewer layers of sclerenchyma cells on the adaxial side and shortened plant height at V2 stage (Supplemental Fig. S4, B-D). Collectively, the evidence from these lines suggest that $\mathrm{ZmBEH} 1$ plays a significant role in leaf angle formation in maize. 
In order to identify the partners that interact with $\mathrm{ZmBEH}$, we screened the B73 leaf cDNA yeast two hybrid library using ZmBEH1 as a bait. A protein that has high homology with OsBZR1 and AtBZR1/BES1 (Supplemental Fig. S1) was identified to have strong interaction with ZmBEH1 (Fig. 3A), which we named as ZmBZR1.

To further confirm the interaction between ZmBZR1 and ZmBEH1 in vivo, we then performed the split luciferase assay with ZmBEH1 fused to the $\mathrm{N}$ terminus of LUC (ZmBEH1-nLUC) and ZmBZR1 fused to the C terminus of LUC (cLUC-ZmBZR1). When the Agrobacterium strain GV3101 containing both the ZmBEH1-nLUC and cLUC-ZmBZR1 constructs was injected into the tobacco leaf, a strong luciferase signal was detected, suggesting that the interaction between ZmBEH1 and ZmBZR1 also occurred in vivo (Fig. 3B).

ZmBZR1 Mu-insertion mutants and CRISPR lines showed reduced leaf angle

To study its function, we obtained two potential loss-of-function mutants of ZmBZR1. One has a uniform Mu inserted to the first exon of ZmBZR1 and the other one has $\mathrm{Mu}$ inserted to the first intron, which were referred to as bzr1-1 and bzr1-2, respectively (Fig. 4A, Supplemental Fig. S3). We found that the expression of $Z m B Z R 1$ in both mutant alleles was significantly decreased (Fig. 4B), and the plants exhibit the erect leaf phenotype as beh1-1. Compared with the WT, the leaf angle of bzr1-1 and bzr1-2 decreased about 27.5\% and 31.3\% at V2 and V10 stage, respectively (Fig. 4D). Corresponding to this, the margin width and area of ligular region decreased in both bzrl-1 and bzr1-2 mutants (Fig. 4E), so does the sclerenchyma layers at adaxial side (Fig. 4F).

At V10 stage, the bzrl-1 reached about $81.8 \%$ and bzr $1-2$ reached about $89.3 \%$ of the WT height (Fig. 4C). However, when compared another nine important agronomic traits, including ear height, leaf length, leaf width and tassel branch number between bzrl and the WT plants at blister stage 
were much more dramatic than that in bzr1-2, indicating that the Mu inserted in the first intron of $Z m B Z R 1$ caused a week allele.

In addition, another two mutant alleles of ZmBZRl (bzrl-3 and bzrl-4)

which presented by the $42 \mathrm{bp}$ and $72 \mathrm{bp}$ deletion in the first exon, were generated through CRISPR/Cas9 system (Supplemental Fig. S6A). The reduced leaf angle and shortened plant height were consistent with the phenotype of bzr1-1 (Supplemental Fig. S6B).

\section{ZmBEH1 and ZmBZR1 act synergistically to regulate the leaf angle}

We suspected whether ZmBZR1 and ZmBEH1 work redundantly in regulating the maize leaf angle. To test this, we created double mutants of bzrl-1 beh1-1 and bzr1-2 beh1-1, and measured the leaf angle at V10 stage. Compared with the single mutant plants, the leaf angle in the double mutants decreased around $6^{\circ}$ and $5^{\circ}$ in bzrl-1 behl-1 and bzrl-2 behl, respectively

(Fig. 5A). Consistently, double mutant plants have upright leaf angle, a narrower margin width and smaller ligular area, resulting in reduced auricle size at V10 stage (Fig. 5B). Except these, the double mutants were almost identical to the single mutants in terms of other agronomic traits e.g., leaf length and width (Supplemental Fig. S7, A and B). Together with the observation that ZmBZR1 and ZmBEH1 physically interact, we propose that they might co-operate with each other to regulate leaf angle.

\section{ZmLG1 regulates the expression of $\mathrm{ZmBZR1}$}

Since ZmLG2 directly regulates the expression of $\mathrm{ZmBEH1}$, we wondered whether ZmLG2 could also regulate the ZmBZR1 as well. However, there was no ZmLG2 ChIP-seq binding site in the ZmBZR1 promoter, and we could not detect the binding of ZmLG2 in $\mathrm{Y} 1 \mathrm{H}$ and dual-luciferase assay. Motif analysis of ZmBZR1 promoter identified an GTAC element, which is the binding motif of well-known transcription factor ZmLG1 controlling leaf angle (Tian et al., 2019; Moreno et al., 1997).

We then tested whether ZmLG1 could regulate the expression of ZmBZR1. 
254 Both Y1H and EMSA assay confirmed a direct binding of ZmLG1 in ZmBZR1 promoter (Fig. 6, A and B). Next, dual-LUC transient assay performed in maize protoplasts confirmed that the ZmLG1 could activate the expression of $Z m B Z R 1$, as the ZmLG2 protein significantly induced the expression of LUC driven by the $Z m B Z R 1$ promoter (Fig. 6C). Furthermore, we conduct the transient expression assay in tobacco (Nicotiana tabacum) leaves. The -640 to -168 sequence in front of TSS of ZmBZR1 promoter was used to drive the expression of $L U C$ gene. The strong LUC signals were detected only when the ZmLG1 protein was co-injected with the ZmBZR1 promoter further validate the activation of ZmLG1 on the expression of ZmBZR1 (Fig. 6D).

We also performed RNA-seq for the $\lg 1$ mutant, and found that the expression of $Z m B Z R 1$ was significantly reduced in the ligular region in $\lg 1$ mutant than those in the WT (unpublished data, Supplemental Fig. S2B), especially in the middle stage of ligule expansion (stage 3 to 4), further indicate the positive regulation of ZmLG1 on the expression of ZmBZR1 (Fig. $6 \mathrm{E})$.

In addition, we generated the $\lg 1$ bzrl-1 and $\lg 1$ bzrl-2 double mutants to test the relationship between ZmLG1 and ZmBZR1, genetically. At the bladesheath boundary, $\lg 1$ bzrl-1 and $\lg 1$ bzrl-2 have no ligule and auricle, which exhibit erect leaf architecture consistence with $\lg 1$ (Supplemental Fig. S8), further indicates the epistatic effect of ZmLG1 on ligule and leaf angle 276 formation.

Yeast one-hybrid identified $Z m S C L 28$ as a downstream target of ZmBZR1 and ZmBEH1

To study how these transcription factors regulate leaf angle, we used yeast one-hybrid to find their potential downstream targets. Y1H assay showed that ZmSCL28 promoter can be targeted by both ZmBZR1 and ZmBEH1 (Fig.

282 7A). ZmSCL28 encodes a GRAS domain transcription factor that has high 283 homolog with rice $D L T$ (Supplemental Fig. S9). In rice, OsBZR1 binds to 
CGTGCG elements (named BRRE motif) in the promoter of DLT gene to suppress its expression, and the similar regulation has also been reported in Arabidopsis (Li and Jin, 2007; Tong et al., 2009).

Next, we obtained a ZmSCL28 uniform $\mathrm{Mu}$ insertion line (scl28-1) and confirmed the insertion in the first exon by PCR (Fig. 7B; Supplemental Fig. S3). The plant architecture of $s c l 28-1$ is similar to that of bzrl-1, bzrl-2 and beh1-1, conferring erect leaves, short margin width, small auricle size and decreased plant height, but the leaf angle is even smaller (Fig. 7, C, D and E; Supplemental Fig. S10, A and C).

To further confirm this, we generated a second loss-of-function mutant of SCL28 by CRISPR/Cas9 (Fig. 7B). This scl28-2 line has a 49 bp deletion located $50 \mathrm{bp}$ downstream of the start codon. The phenotype of $s c l 28-2$ is similar to scl28-1, e.g., small leaf angle, upright leaf and short plant height (Fig. 7, C, D and E; Supplemental Fig. S10, A and C), confirming that ZmSCL28 also plays important roles in controlling plant architecture.

To test the relationship among ZmSCL28, ZmBZR1 and ZmBEH1 genetically, the double mutants of bzr1-1 scl28-1, bzr1-2 scl28-1 and beh1-1 scl28-1 were generated. The double mutants had essentially the same phenotype as the scl28-1 single mutant regarding plant height, leaf angle, auricle size, leaf length and width, with an exception being that the leaves of bzr1-1 scl28-1 were slightly wider than that of scl28-1 (Fig. 7, D and E; Supplemental Fig. S10, B and C). All these results support the conclusion that ZmSCL28 is the direct target of ZmBZR1 and ZmBEH1 to regulate the leaf angle in maize.

\section{DISCUSSION}

Increasing planting density is one of the key strategies to increase crop yield, and the selection of small leaf angle that determine the upright leaf 311 architecture contribute significantly to the dense planting in maize (Tian et al., 2011; Pan et al., 2017; Wei et al., 2018; Tian et al., 2019; Zhao et al., 2019). ZmLG1 and ZmLG2 are two key loci that control the ligule 
development and then influence leaf angle formation, however, mutations at these two loci cause the abolish of normal ligule, which makes it easier for water to permeate into the leaf sheath, causing bacterial and fungal infections. In addition, the extremely erect leaf angle from the introgression lines of these alleles also limits their use in the commercial crop production. Therefore, we believe it is important to explore the downstream targets of ZmLG1 and ZmLG2 to achieve both small leaf angle and a normal ligule development.

To do so, we first examined the target of ZmLG2 and found ZmBEH1, a BZR1/BES1 homolog, and validate its role in controlling plant architecture. Our RNA-seq studies showed that ZmLG2 could regulate leaf angle through $\mathrm{ZmBEH1}$ in the stage of ligule/auricle expansion since the expression of ZmBEH1 peaked at this stage (Fig.1D). The loss of function of ZmBEH1 exhibit semi-dwarf phenotype, and the leaf angle was significantly decreased (Fig. 2, C and D). This decreasing may be caused by the reduced number of sclerenchyma cell layers at the adaxial site of the ligular region (Fig. 2F). However, how $\mathrm{ZmBEH1}$ regulates the sclerenchyma cell development on adaxial /abaxial sites needs further exploration.

Interestingly, Y2H and split LUC assay showed that ZmBEH1 could physically interact with ZmBZR1. The function of BZR1 has been well studied in Arabidopsis and rice, which plays central roles in brassinosteroid signaling pathways to affect plant development (i.e., erect leaves, phloem, and xylem differentiation), abiotic stress and so on (Wang et al., 2012; Sun et al., 2015; Saito et al., 2018; Min et al., 2019). The ZmBZR1 mutant phenotypes were similar to that of $\mathrm{ZmBEH1}$, e.g., semi-dwarf and decreased leaf angle (Fig. 4), however, the function of these two BES1/BZR1 family numbers were not redundantly at least in regulating the leaf angle formation, since the bzr1-1 beh1-1 and bzr1-2 beh1-1 double mutants displays smaller leaf angle than any of the single mutant (Fig. 5). We also found that ZmLG1, but not ZmLG2, could directly bind to the $Z m B Z R 1$ promoter and active its 
expression (Fig. 6). The distinct regulation of ZmLG1 and ZmLG2 on $Z m B Z R 1$ and $Z m B E H 1$ indicates a complex regulation specificity of ZmLG1 and $\mathrm{ZmLG} 2$ on the BR signaling factors, which further controls the maize leaf angle formation.

Next, we identified ZmSCL28 as a direct downstream target of ZmBZR1 and ZmBEH1 through Y1H assay. ZmSCL28 is the homolog gene of DLT in rice, the mutation of $Z m S C L 28$ shared similar phenotypes with $d l t$, e.g. dwarf and erect leaves, indicating the conserved function of these TFs in the different species. In rice, $D L T$ was bind by OsBZR1 in the promoter through the BR-response element (Tong et al., 2009; Tong and Chu, 2009; Tong et al., 2012; Hirano et al., 2017; Qiao et al., 2017) and the loss-of-function mutant $d l t$ was similar to BR-insensitive mutants (Tong et al., 2009). In maize, not only ZmBZR1, but also ZmBEH1 could bind to the BR-response element in the $Z m S C L 28$. We also proved that $Z m S C L 28$ is the downstream target of ZmBZR1 and ZmBEH1 genetically, since the double mutants of bzrl-1 scl28-1, bzrl-2 scl28-1 and beh1-1 scl28-1 have almost identical phenotypes

with scl28-1 single mutant, for example, the leaf angle was $16.6^{\circ}$ in $s c l 28-1$

mutant and $16.1^{\circ}$ in bzrl-1 scl28-1 double mutant, however, the angle size

was much larger in that of single mutants of bzrl-1 $\left(25.7^{\circ}\right)$, bzrl-2 $\left(26.5^{\circ}\right)$

and beh-1 $\left(26.6^{\circ}\right)$ (Fig. 7).

Taken together, we propose a ZmLG2-BEH1-SCL28 regulatory cascade that effects leaf angle formation in maize (Fig. 8). In this model, we showed that $\mathrm{ZmBEH1}$ is directly activated by the $\mathrm{ZmLG}$, and $\mathrm{ZmBEH} 1$ can interact with ZmBZR1. ZmBZR1 is directly activated by the ZmLG1, another wellknown TF plays important roles in determine leaf architecture. Furthermore, we found that both ZmBEH1 and ZmBZR1 bind to the ZmSCL28 promoter to regulate leaf angle. As the downstream targets of $\mathrm{ZmLG1}$ or $\mathrm{ZmLG}$, the loss of function mutants of $Z m B E H 1, Z m B Z R 1, Z m S C L 28$ have normal 
372 development of leaf ligule as expected, however, the size of leaf angle was

373 dramatically reduced. Therefore, we propose that these three novel target loci,

$374 \mathrm{ZmBZR} 1, \mathrm{ZmBEH} 1$ and ZmSCL28 are ideal targets to manipulate leaf angle in

375 order to generate upright and semi-dwarf plant architecture, the two important

376 traits pursued by breeders to increase the planting density and yields.

\section{Materials and Methods}

\section{Plant Materials and Growth Conditions}

Uniform Mu mutants with the stock number UFMu-13537, UFMu-03258, UFMu-13557 were requested from the maize genetic cooperation stock center. After two generations of backcrossing to maize inbred lines W22, the $\mathrm{BC}_{2} \mathrm{~F}_{1}$ population that segregating the WT and mutant phenotypes were obtained. The stable single mutants from each gene were crossed to each other to obtain the double mutant.

To observe the phenotypes in seedling stage, seeds were planted into soil and were grown in a growth room with the setting of $28^{\circ} \mathrm{C}, 12$-h-light $/ 22^{\circ} \mathrm{C}$, 12-h-dark cycle and $60 \%$ relative humidity.

\section{Yeast one-hybrid}

To test the binding of ZmLG1 and ZmLG2 to the promoter of ZmBZR1 or $\mathrm{ZmBEH1}$ in the yeast one-hybrid assay (Y1H), the full-length CDS of ZmLG1, ZmLG2 were amplified and cloned into the pJG4-5 vector respectively to generate the destination plasmids. The fusion plasmids carrying the target genes were co-transformed with the Lac $Z$ reporter vector $(\mathrm{LacZi}-2 \mu)$ driven by the promoter of $Z m B Z R 1$ or $Z m B E H 1$ into the yeast strain EGY48. The empty vector pJG4-5 and the Lac $Z$ reporter driven by the promoter of ZmBZR1 or ZmBEH1were co-transformed as negative control.

Similarly, to test the binding of ZmBZR1 and ZmBEH1 to the ZmSCL28 promoter, the full-length CDS of $Z m B Z R 1, Z m B E H 1$ were amplified and cloned into the pJG4-5 vector and the fusion plasmids were co-transformed with the $L a c Z$ reporter vector $(\mathrm{LacZi}-2 \mu)$ driven by the ZmSCL28 promoter. 
promoter served as negative control.

The transformed yeast stains were plated on SD/-Trp-Ura medium and grown at $30^{\circ} \mathrm{C}$ for 3 days. After that, the transformants were tested on SD screening medium containing X-gal (5-bromo-4-chloro-3-indolyl-b-Dgalactopyranoside) at $30^{\circ} \mathrm{C}$ for 4 days. The sequences of primers used in $\mathrm{Y} 1 \mathrm{H}$ were listed in the Supplemental Table 1.

\section{Transient expression assay in maize protoplast}

For the dual-luciferase transient expression assay, ZmBZRI and ZmBEHI promoter segments (808 bp and $1290 \mathrm{bp}$, respectively) were amplified from inbred line B73 and recombined into pGreenII 0800-LUC vector to generate the pZmBZR1/BEH1::LUC and pZmSCL28::LUC plasmids as reporter. The renillia luciferase $(\mathrm{REN})$ gene driven by $35 \mathrm{~S}$ promoter in the pGreenII 0800LUC vector was used as the internal control. The full-length CDS of ZmLG1, ZmLG2 were amplified and recombined into $\mathrm{pHW}-3 \mathrm{xAvi}$ vector via Gateway LR Clonase II Enzyme mix driven by the $35 \mathrm{~S}$ promoter, forming the effectors. The empty pHW-3 $\times$ Avi vector was used as control. The transient dualluciferase assays were performed in the maize protoplasts collected from the leaves of 12-day-old etiolated seedling of inbred line B73. The luciferase signal was detected using dual-luciferase assay reagents following the manufacturer's instructions. Relative LUC activity was calculated by normalizing LUC activity to REN activity. The sequences of primers used in the transient expression assay are listed in Supplementary Table S1.

\section{Transient expression assay in Nicotiana benthamiana leaves}

The luciferase intensity assay was performed to examine the interactions between protein and promoter sequence. The full-length CDS of ZmLG1/LG2 and ZmBZR1/BEH1 was amplified and recombined into pCAMBIA1300-35s-GFP forming the effectors. ZmSCL28 promoter (1048 bp) also recombined into pGreenII 0800-LUC vector with minimal CaMV $35 \mathrm{~S}$ promoter. Then these constructs were transformed into the Agrobacterium strain GV3101. Next, Agrobacteria was infiltrated alone or 
432 together into the leaves of tobacco plants. About $3 \mathrm{~d}$ after infiltration, the

433 transformed tobacco leaves were observed by Nightshade LB 985.All images

434 were generated from LB985-Berthold.

\section{Electrophoretic Mobility Shift (EMSA) Assay}

436 EMSA assay was performed as described previously (Song et al., 2020).

437 The protein fused with GST has been purified firstly. The full-length

438 BZR1/BEH1 cDNA was amplified with gene-specific primers and cloned

439 into expression vector Pcold-GST. The construct was transformed into

440 Escherichia coli BL21 cells, grown at $37^{\circ} \mathrm{C}$. At $\mathrm{OD}_{600}=0.4-0.5$, the culture

441 solution was placed at $16^{\circ} \mathrm{C}$ and let stand for 30 minutes. Isopropylthio- $\beta$ -

442 galactoside was added at the final concentration of $1 \mathrm{mM}$, and the culture was

443 incubated at $16^{\circ} \mathrm{C}$ for 24 hours. The Pcold-GST-BZR1/BEH1 expressed

444 GST-BZR1/BEH1 fusion protein was purified with BeaverBeads GSH

445 (Beaver, Suzhou, China) and used for EMSA. The probes with biotin were 446 synthesized according to manufacturer's recommendation (Supplementary

447 Table S1). The affinity bands labeled with biotin probes were detected by

448 EMSA kit (Beyotime Biotechnology) and approximately $10 \mathrm{~nm}$ of probe was

449 used for each binding assay. For competition assay, unlabeled probe was

450 added to the reactions to detect the binding specificity.

451 Yeast two-hybrid assays

452 The Yeast two-hybrid assay was performed using the Matchmaker ${ }^{\text {TM }}$ Gold 453 Yeast Two-Hybrid System (Clontech). The full-length coding region of 454 ZmBZR1 were ligated into pGBKT7 plasmid as bait and the full-length 455 coding regions of $\mathrm{ZmBEH} 1$ was fused into pGADT7 vector as prey. The bait and prey constructs were co-transformed into $\mathrm{Y} 2 \mathrm{H}$ Gold yeast strain and grow at $30^{\circ} \mathrm{C}$ for 3 days. Next, the transformants were tested on SD screening medium containing X- $\alpha-\mathrm{Gal}$ (5-Bromo-4-chloro-3-indoxyl- $\alpha-\mathrm{D}$ galactopyranoside) at $30^{\circ} \mathrm{C}$ for $4 \mathrm{~d}$. Empty vectors were co-transformed as

460 negative controls. The sequences of primers used in $\mathrm{Y} 2 \mathrm{H}$ were listed in the 461 Supplemental Table 1. 


\section{The split luciferase complementation assay}

The split firefly luciferase complementation assay was performed to examine the interactions between ZmBEH1 and ZmBZR1 using the constructs nLUC and cLUC. The full-length CDS of ZmBZRl without the stop codon and $\mathrm{ZmBEH} 1$ with the stop codon were amplified and cloned into nLUC and cLUC, respectively, forming the nLUC-ZmBEH1 and ZmBZR1cLUC constructs. Agrobacterium strain GV3101 cells carrying all constructs were transiently infiltrated into Nicotiana benthamiana leaves. After culturing for three days, luciferin $(1 \mathrm{mM})$ was sprayed to activate luciferase, and the fluorescence signals were observed by the Chemiluminescent Imaging System (Tanon-5200). The primers used in the split firefly luciferase complementation assays are listed in Supplemental Table 1.

\section{Phylogenetic analysis of ZmBZR1/BEH1 and ZmSCL28}

The full-length amino acid sequences of ZmBZR1/BEH1 and ZmSCL28 were used to BLAST search in protein databases of GRAMENE (http://www.gramene.org/), TAIR (http://www.arabidopsis.org) and RICEDATE (http://www.ricedata.cn/gene/) to identify the homologous genes in maize, Arabidopsis and rice. The amino acid sequences were aligned using the clustalw-2.0.10 software. A phylogenetic tree was constructed based on this alignment result using the neighbour-joining method in MEGA version 6 with the following parameters: Poisson correction, pairwise deletion, uniform rates and bootstrap (1,000 replicates).

\section{Statistical Analysis}

To determine statistical significance, we employed Student's test. ( ${ }^{*} \mathrm{P}<$ $0.05, * * \mathrm{P}<0.01)$

\section{RNA extraction and $q P C R$ analysis}

Total RNA was extracted using TRIzol reagent (Ambion) from maize leaves. The RNA samples were treated with DNase I (Thermo) and the concentration was measured by DS-11. Subsequently, cDNA was prepared 
using M-MLV Reverse Transcriptase and qRT-PCR were conducted using the

492 SYBR Premix Ex Taq kit (Trans) on a Step One System (Applied 493 Biosystems). The quantification method $\left(2^{-\Delta \mathrm{Ct}}\right)$ was used and the gene expression level was estimated using three independent biological replicates. The maize Ubi2 (UniProtKB/TrEMBL, Q42415) gene was used as an internal control to normalize the data. The process of qRT-PCR consisted of an initial denaturation step at $95{ }^{\circ} \mathrm{C}$ for $10 \mathrm{~min}$, followed by 40 cycles at $95{ }^{\circ} \mathrm{C}$ for $15 \mathrm{~s}$ and $60{ }^{\circ} \mathrm{C}$ for $30 \mathrm{~s}$. The primers for qRT-PCR are shown in Supplemental Table S1.

\section{Transformation}

The CRISPR/Cas9 constructs for ZmBEH1, ZmBZR1 and ZmSCL28 were generated using a previously reported vector pBUE411 (Xing et al., 2014). The specific target sites and PAMs (the last three nucleotides) of $Z m B E H 1$, ZmBZR1 and ZmSCL28 were designed and cloned into pBUE411 vector. These constructs were introduced into the Agrobacterium tumefaciens strain EHA105 and transformed into the maize inbred line KN5585 in WIMI Biotechnology Co., Ltd. The $\mathrm{T}_{1}$ plant was self-crossed to generate $\mathrm{T}_{2}$ plants for further research. The mutated sequences of $\mathrm{T}_{2}$ plants were confirmed by sequencing. The primers were shown in Supplementary Table S1.

\section{ACCESSION NUMBERS}

Sequence data from this article can be found in Maize GDB Database under the following accession numbers: ZmLG1 (Zm00001d002005), ZmLG2 (Zm00001d046305), ZmSCL28 (Zm00001d045507).

\section{ACKNOWLEDGMENTS}

We thank the maize genetics cooperation stock center for kindly providing uniform $\mathrm{Mu}$ mutants with the stock number UFMu-13537, UFMu-03258, UFMu-13557. We thank Prof. Sarah Hake (University of California, Berkeley) for offering seeds of $\lg 1$ and $\lg 2$ mutants. We would also like to thank Prof. Feng Tian (China Agricultural University) for sharing the 
521 construct to induce the ZmLG1 recombinant proteins and Prof. Qijun Chen

522 (China Agricultural University) for sharing the plasmid pBUE411.

\section{Figure legends}

524 Figure 1. ZmLG2 directly regulates the expression of $\mathrm{ZmBEH}$. A, ChIP-Seq shows ZmLG2 binds to the ZmBEH1 promoter. B, ZmLG2 directly binds to the ZmBEH1 promoter in EMSA. "-" indicates the absence of proteins. "+" indicate the present; FP indicates free probe. C, Dual-luciferase assay shows relative transactivation of $\mathrm{ZmLG} 2$ to $\mathrm{ZmBEH1}$ promoter. The coding sequence of ZmLG2 driven by the $35 \mathrm{~S}$ promoter was used as an effector, and the empty vector was used as an effector control in transient Luciferase assay performed in maize protoplasts. The vector with the Renilla reniformis (REN) gene driven by a $35 \mathrm{~S}$ promoter and firefly luciferase (LUC) gene driven by promoter sequence from $\mathrm{ZmBEH} 1$ were used as the reporter. The LUC/REN ratio represents the relative activity of the promoters. Error bars represent SD $(\mathrm{n}=5)$ *represents $\mathrm{P}<0.05$ determined by Student's t test. D, RNA-Seq data shows the expression profiles of $\mathrm{ZmBEH1}$ in different maturation stage of the ligular region (S0 to $\mathrm{S} 4$ ) in WT and $\lg 2$ mutant. Details showed in Supplemental Fig. S2, A and C.

Figure 2. Phenotypes of beh1-1 plants. A, Uniform Mu-mediated insertion of ZmBEH1. A maize transposon-insertion line behl-1 was obtained from the maize Uniform $\mathrm{Mu}$ resource, which carried a Mu insertion (UFMu-13557) in the 5'-UTR of ZmBEH1. B, Expression of ZmBEH1 gene in WT inbred line W22 and beh1-1 plants at V2 stage. **represents $\mathrm{P}<0.01$ determined by Student's t test. C, Plant height of WT inbred line W22 and beh1-1 plants at V2 and V10 stages. From $\mathrm{C}$ to $\mathrm{E}, *$ represents $\mathrm{P}<0.05$, **represents $\mathrm{P}<0.01$ determined by Student's t test, $\mathrm{n}=20$. D, Leaf angle changes between WT inbred line W22 and beh1-1 plants at V2 and V10 stages. E, Quantitative measurements of leaf ligule margin width and ligular area in WT inbred W22 and beh1-1 plants at 15-day-old seedling stage. F, Cross-sections of the ligule 
from WT inbred line W22 and beh1-1 plants at V2 stage. The sclerenchyma cell (SC) layers stained red with safranin. Number of SC cell files at the adaxial and abaxial side were calculated from 10 replicates. Error bars are SD. $* * \mathrm{P}<0.01$. Scale bars, $100 \mu \mathrm{m}$.

Figure 3. ZmBEH1 interacts with ZmBZR1. A, Interaction between ZmBZR1 and ZmBEH1 in yeast two-hybrid assays. Different concentrations of co-transformed yeast cells were spotted on synthetic dropout (SD) medium without tryptophan and leucine (SD/-T-L), or without tryptophan, leucine, histidine and adenine, and plus $20 \mathrm{mg} / \mathrm{mL} \mathrm{X}$ - $\alpha$-gal (SD/-T-L-H-A + X- $\alpha$-gal). $\mathrm{B}$, Interaction between ZmBZR1 and ZmBEH1 in the split luciferase assay.

Figure 4. Characterization of bzrl-1 and bzrl-2 mutants. A, Gene model shows the inserted position of Uniform Mu (UFMu-13537 and UFMu-03258) in the $1^{\text {st }}$ exon $(b z r 1-1)$ and $1^{\text {st }}$ intron (bzrl-2) of ZmBZRl gene. B, Expression analysis of ZmBZR1 in WT inbred line W22 and bzrl-1, bzrl-2 plants at V2 stage. C, Plant height of WT inbred line W22 and bzrl-1, bzrl2 plants in V2 and V10 stages. Different letters above the columns indicate statistically significant differences between groups $(n=20)$. D, Leaf angle changes between WT inbred line W22 and bzrl-1, bzrl-2 plants in V2 and V10 stages $(n=20)$. E, Quantitative measurements of leaf ligule margin width and ligular area in WT inbred line W22 and bzrl-1, bzrl-2 plants at V2 and V10 stages $(n=20)$. Different letters above the columns indicate statistically significant differences between groups. F, Cross-sections of the ligular region from WT inbred line W22 and bzrl-1, bzrl-2 plants at V2 stage. The sclerenchyma cell (SC) layers stained red with safranin. Number of SC cell files at the adaxial and abaxial side were calculated from 10 replicates. Error bars are SD. Scale bars, $100 \mu \mathrm{m}$.

Figure 5. Leaf angle observation from single and double mutants of bzrl and beh1. A, Leaf angle morphology of WT inbred line W22, single mutants of bzrl-1, bzrl-2 and beh1-1, double mutants of bzrl-1 beh1-1 and bzrl-2 beh1- 
1. B, Quantitative measurements of leaf angle $(n=20)$, margin width $(n=10)$ and ligular area $(n=10)$ in W22, single and double mutants. Different letters above the columns indicate statistically significant differences between groups.

Figure 6. ZmLG1 directly activate the expression of ZmBZR1. A, ZmLG1 directly binds to the ZmBZR1 promoter in yeast one hybrid (Y1H) assay. B, ZmLG1 directly binds to the ZmBZR1 promoter in EMSA. "-" indicates the absence of proteins. "+" indicate the present; FP indicates free probe. C, ZmLG1 actives the promoter of ZmBZR1 in Dual-luciferase assay. The coding sequence of LG1 driven by the 35S promoter was used as an effector, and the empty vector was used as an effector control in transient luciferase assay performed in maize protoplasts. The vector with the Renilla reniformis (REN) gene driven by a $35 \mathrm{~S}$ promoter and firefly luciferase (LUC) gene driven by promoter sequence from $Z m B Z R 1$ were used as the reporter. The LUC/REN ratio represents the relative activity of the promoters. Error bars represent $\mathrm{SD}(\mathrm{n}=5) * *$ represents $\mathrm{P}<0.01$ determined by Student's t test. $\mathrm{D}$, Transient luciferase intensity assay performed in tobacco leaves showing that ZmLG1 actives the promoter of ZmBZR1. E, RNA-Seq data shows the expression of $Z m B Z R 1$ in different maturation stage of ligular region (S0 to S4) in WT and $\lg 1$ mutant. Details showed in Supplemental Fig. S2.

Figure7. ZmSCL28 works downstream of ZmBZR1 and ZmBEH1. A, Y1H assays showed that ZmBZR1 and ZmBEH1 bound to the promoter of ZmSCL28. B, Gene model presents the positions of two mutant alleles in ZmSCL28. The triangle shows the position of a Mu insertion (UFMu-09491) from line scl28-1 at W22 genetic background, and red line shows the position of a single-guide RNA (sgRNA) designed to mutate the ZmSCL28 using the CRISPR/Cas9 technology. The sequence of sgRNA is highlighted in blue, and the symbols "..." indicate the deletion caused by CRISPR/Cas9-induced mutations in line scl28-2 at KN5585 genetic background. C, Leaf angle morphology in the WT inbred line W22 and mutant scl28-1, WT inbred line 
KN5585 and mutant scl28-2 at V10 stage. Scale bars $=5 \mathrm{~cm}$. D, Leaf angle morphology in the WT W22 and double mutants of bzrl-1 scl28-1, bzrl-2 scl28-1 and beh1-1 scl28-1 at V10 stage. Scale bars = $5 \mathrm{~cm}$. E, Quantitative measurements of leaf angle $(n=20)$, margin width $(n=10)$ and ligular area $(\mathrm{n}=10)$ in single and double mutants. Different letters above the columns indicate statistically significant differences between groups.

Figure 8. A proposed ZmLG2 (LG1)-ZmBEH1 (BZR1)-ZmSCL28 module regulates leaf angle in maize. The $\mathrm{ZmLG} 2$ binds to the protomer of $\mathrm{ZmBEH1}$ to activates its expression. The ZmBEH1 interact with ZmBZR1, which is a direct target of $\mathrm{ZmLG}$, to regulate the expression of a downstream target gene, ZmSCL28. These proposed regulatory cascades may control the leaf angle size in maize.

Supplemental Table S1. The list of primers used in this paper.

Supplemental Fig. S1. Phylogenetic analysis of BZR1/BES1 family proteins in Maize, Rice and Arabidopsis.

Supplemental Fig. S2. Tissues of the ligular region collected from five different maturation stages of $\lg 2(\mathrm{~A})$ or seven of $\lg 1(\mathrm{~B})$.

Supplemental Fig. S3. PCR identification of beh1-1, bzrl-1, bzrl-2 and scl28-1 mutants.

Supplemental Fig. S4. Phenotypes of beh1-1 mutants.

Supplemental Fig. S5. Agronomic characters of bzrl-1 mutant.

Supplemental Fig. S6. Characterization of the CRISPR/Cas9-mediated knock-out mutants of $Z m B Z R 1$.

Supplemental Fig. S7. Phenotypes of the single and double mutants of bzrl1, bzrl-2 and beh1-1.

Supplemental Fig. S8. Phenotypes of single mutants and $\lg 1$ bzrl-1, $\lg 1$ bzrl-2 double mutant plants.

636 Supplemental Fig. S9. Phylogenetic analysis of SCL family.

637 Supplemental Fig. S10. Phenotypes of scl28-1/scl28-2 and bzrl-1 scl28-1, 


\section{Literature Cited}

640

641

642

643

644

645

646

647

648

649

650

651

652

653

654

655

656

657

658

659

660

661

662

663

664

665

666

667

668

669

670

671

672

673

674

675

676

677

678

679

680

681

682

683

684

685

686

687

688

689

Bai MY, Zhang LY, Gampala SS, Zhu SW, Song WY, Chong K, Wang ZY (2007) Functions of OsBZR1 and 14-3-3 proteins in brassinosteroid signaling in rice. Proc Natl Acad Sci USA 104: 13839-13844

Bolduc N, O'Connor D, Moon J, Lewis M, Hake S (2012) How to pattern a leaf. Cold Spring Harb Symp Quant Biol 77: 47-51

Cao Y, Zeng H, Ku L, Ren Z, Han Y, Su H, Dou D, Liu H, Dong Y, Zhu F, Li T, Zhao Q, Chen Y (2020) ZmIBH1-1 regulates plant architecture in maize. J Exp Bot 71: 2943-2955

Chen LG, Gao Z, Zhao Z, Liu X, Li Y, Zhang Y, Liu X, Sun Y, Tang W (2019) BZR1 family transcription factors function redundantly and indispensably in BR signaling but exhibit BRI1-independent function in regulating anther development in Arabidopsis. Mol Plant 12: $1408-1415$

Duvick DN (2005) Genetic progress in yield of United States maize (Zea mays L.). Maydica: 193202

Muehlbauer GJ, Fowler JE, Girard L, Tyers R, Harper L, Freeling M (1999) Ectopic expression of the maize homeobox gene liguleless 3 alters cell fates in the leaf. Plant Physiol 119: 651-662

Harper L, Freeling M (1996) Interactions of liguleless 1 and liguleless2 function during ligule induction in maize. Genetics 144: 1871-1882

Hirano K, Yoshida H, Aya K, Kawamura M, Hayashi M, Hobo T, Sato-Izawa K, Kitano H, Ueguchi-Tanaka M, Matsuoka M (2017) Small organ size 1 and small organ size 2/dwarf and low tillering form a complex to integrate auxin and brassinosteroid signaling in rice. Mol Plant 10: 590-604

Kir G, Ye H, Nelissen H, Neelakandan AK, Kusnandar AS, Luo A, Inzé D, Sylvester AW, Yin Y, Becraft PW (2015) RNA interference knockdown of BRASSINOSTEROID INSENSITIVE1 in maize reveals novel functions for brassinosteroid signaling in controlling plant architecture. Plant Physiol 169: 826-839

Ku L, Wei X, Zhang S, Zhang J, Guo S, Chen Y (2011) Cloning and characterization of a putative TAC1 ortholog associated with leaf angle in maize (Zea mays L.). PLoS ONE 6: e20621

Lambert RJ, Johnson RR (1978) Leaf angle, tassel morphology, and the performance of maize hybrids. Crop Sci 18: 499-502

Li H, Ye K, Shi Y, Cheng J, Zhang X, Yang S (2017) BZR1 Positively Regulates Freezing Tolerance via CBF-Dependent and CBF-Independent Pathways in Arabidopsis. Mol Plant 10: $545-559$

Li JM, Jin H (2007) Regulation of brassinosteroid signaling. Trends Plant Sci 12: 37-41

Luo XM, Lin WH, Zhu S, Zhu JY, Sun Y, Fan XY, Cheng M, Hao Y, Oh E, Tian M, Liu L, Zhang M, Xie Q, Chong K, Wang ZY (2010) Integration of light- and brassinosteroidsignaling pathways by a GATA transcription factor in Arabidopsis. Dev Cell 19: 872-883

Makarevitch I, Thompson A, Muehlbauer GJ, Springer NM (2012) Brdl gene in maize encodes a brassinosteroid C-6 oxidase. PLoS ONE 7: e30798.

Mantilla-Perez MB, Salas Fernandez MG (2017) Differential manipulation of leaf angle throughout the canopy: current status and prospects. J Exp Bot 68: 5699-5717

Moreno MA, Harper LC, Krueger RW, Dellaporta SL, Freeling Michael (1997) liguleless 1 encodes a nuclear-localized protein required for induction of ligules and auricles during maize leaf organogenesis. Genes Dev: 616-628

Min HJ, Cui LH, Oh TR, Kim JH, Kim TW, Kim WT (2019) OsBZR1 turnover mediated by OsSK22-regulated U-box E3 ligase OsPUB24 in rice BR response. Plant J 99: 426-438

Moreno MA, Harper LC, Krueger RW, Dellaporta SL, Freeling M (1997) liguleless 1 encodes a nuclear-localized protein required for induction of ligules and auricles during maize leaf organogenesis. Genes Dev 11: 616-628

Pendleton JW, Smith GE, Winter SR, Johnston TJ (1968) Field investigations of the relationships of leaf angle in corn (Zea mays L.) to grain yield and apparent photosynthesis. Agronomy Journal 60: 422-424

Qiao S, Sun S, Wang L, et al. (2017) The RLA1/SMOS1 transcription factor functions with 
OsBZR1 to regulate brassinosteroid signaling and rice architecture. Plant Cell 29: 292-309

Ren Z, Wu L, Ku L, Wang H, Zeng H, Su H, Wei L, Dou D, Liu H, Cao Y, Zhang D, Han S, Chen Y (2020) ZmILII regulates leaf angle by directly affecting liguleless 1 expression in maize. Plant Biotechnol J 18: 881-883

Saito M, Kondo Y, Fukuda H (2018) BES1 and BZR1 redundantly promote phloem and xylem differentiation. Plant Cell Physiol 59: 590-600

Song Z, Yan T, Liu J, Bian Y, Heng Y, Lin F, Jiang Y, Wang Deng X, Xu D (2020) BBX28/BBX29, HY5 and BBX30/31 form a feedback loop to fine-tune photomorphogenic development. Plant J 104: 377-390

Sun S, Chen D, Li X, Qiao S, Shi C, Li C, Shen H, Wang X (2015) Brassinosteroid signaling regulates leaf erectness in Oryza sativa via the control of a specific U-type cyclin and cell proliferation. Dev Cell 34: 220-228

Tian J, Wang C, Xia J, Wu L, Xu G, Wu W, Li D, Qin W, Han X, Chen Q, Jin W, Tian F (2019) Teosinte ligule allele narrows plant architecture and enhances high-density maize yields. Science 365: 658-664

Tong H, Chu C (2009) Roles of DLT in fine modulation on brassinosteroid response in rice. Plant Signal Behav 4: 438-439

Tong H, Jin Y, Liu W, Li F, Fang J, Yin Y, Qian Q, Zhu L, Chu C (2009) DWARF AND LOW-TILLERING, a new member of the GRAS family, plays positive roles in brassinosteroid signaling in rice. Plant J 58: 803-816

Tong H, Liu L, Jin Y, Du L, Yin Y, Qian Q, Zhu L, Chu C (2012) DWARF AND LOWTILLERING acts as a direct downstream target of a GSK3/SHAGGY-like kinase to mediate brassinosteroid responses in rice. Plant Cell 24: 2562-2577

Tu X, Mejía-Guerra MK, Valdes Franco JA, Tzeng D, Chu PY, Shen W, Wei Y, Dai X, Li P, Buckler ES, Zhong S (2020) Reconstructing the maize leaf regulatory network using ChIP-seq data of 104 transcription factors. Nat Commun 11: 5089

Walsh J, Waters CA, Freeling M (1998) The maize gene liguleless 2 encodesa basic leucine zipper protein involvedin the establishment of the leaf blade-sheath boundary. Genes Dev 12: $208-218$

Wang W, Bai MY, Wang ZY (2014) The brassinosteroid signaling network-a paradigm of signal integration. Curr Opin Plant Biol 21: 147-153

Wang ZY, Bai MY, Oh E, Zhu JY (2012) Brassinosteroid signaling network and regulation of photomorphogenesis. Annu Rev Genet 46: 701-724

Wei H, Zhao Y, Xie Y, Wang H (2018) Exploiting SPL genes to improve maize plant architecture tailored for high density planting. J Exp Bot 20:4675-4688

Xing HL, Dong L, Wang ZP, Zhang HY, Han CY, Liu B, Wang XC, Chen QJ (2014) A CRISPR/Cas9 toolkit for multiplex genome editing in plants. BMC Plant Biol 14: 327

Zhang J, Ku LX, Han ZP, Guo SL, Liu HJ, Zhang ZZ, Cao LR, Cui XJ, Chen YH (2014) The ZmCLA4 gene in the $q L A 4-1$ QTL controls leaf angle in maize (Zea mays L.). J Exp Bot 65: 5063-5076

\section{Zhao Y, Wang H, Bo C, Dai W, Zhang X, Cai R, Gu L, Ma Q, Jiang H, Zhu J, Cheng B} (2018) Genome-wide association study of maize plant architecture using $\mathrm{F}_{1}$ populations. Plant Mol Biol 99:1-15 
A

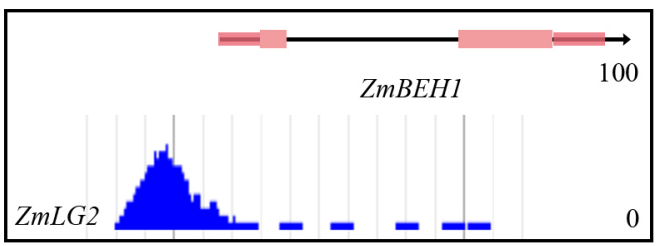

C
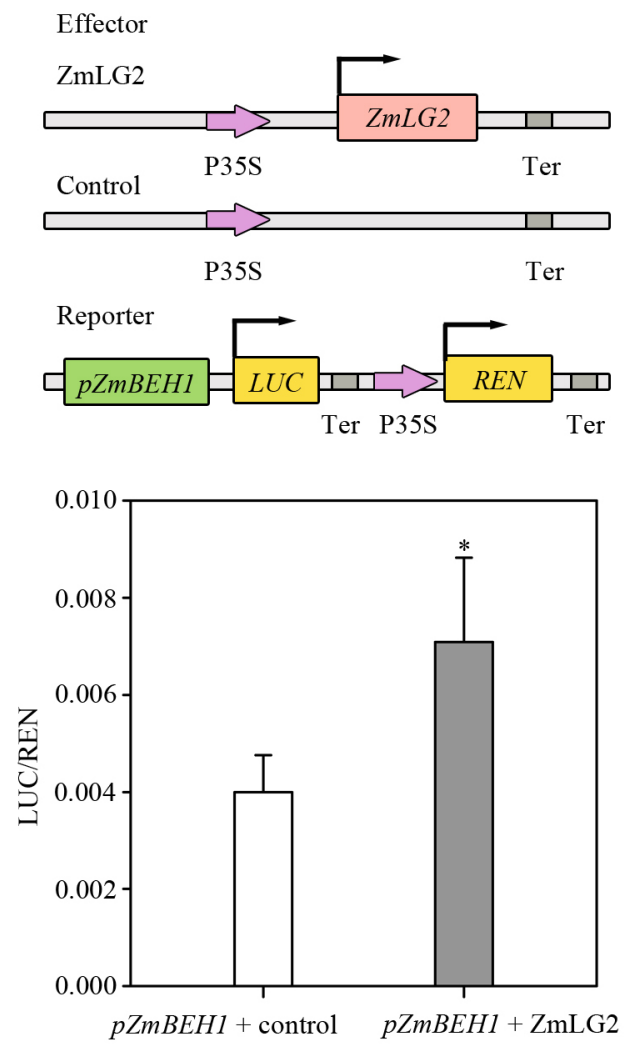

B

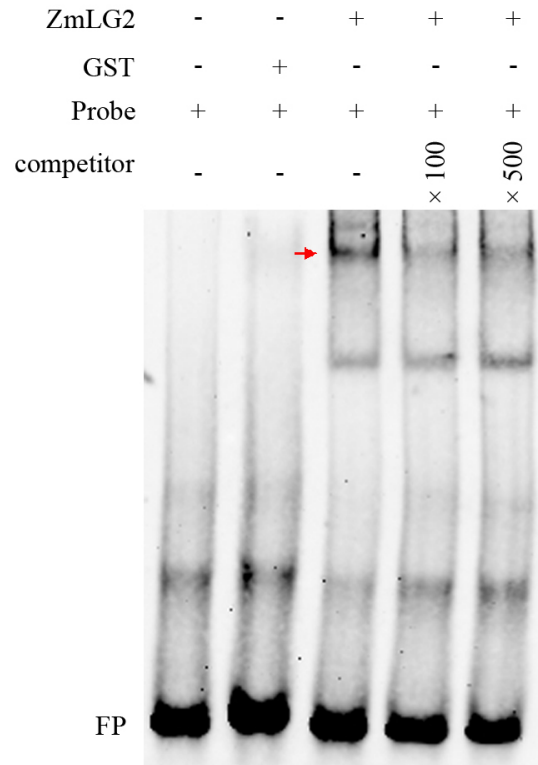

D

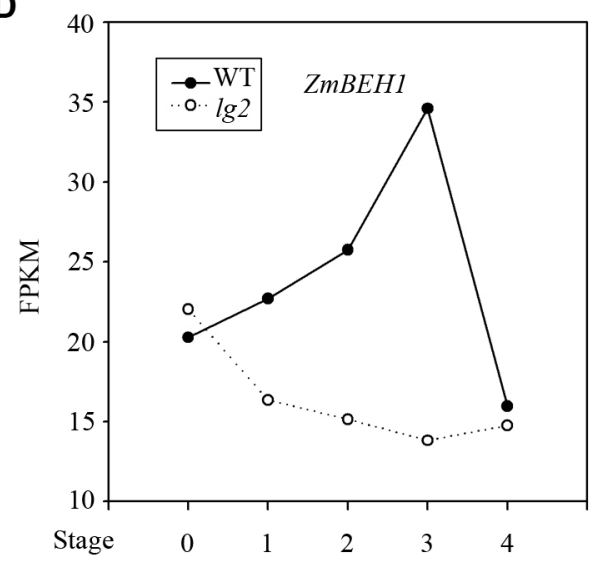

Figure 1. ZmLG2 directly regulates the expression of ZmBEH1. A, ChIP-Seq shows ZmLG2 binds to the ZmBEH1 promoter. B, ZmLG2 directly binds to the ZmBEH1 promoter in EMSA. "-" indicates the absence of proteins. "+" indicate the present; FP indicates free probe. C, Dual-luciferase assay shows relative transactivation of $\mathrm{ZmLG} 2$ to $\mathrm{ZmBEH1}$ promoter. The coding sequence of $\mathrm{ZmLG} 2$ driven by the $35 \mathrm{~S}$ promoter was used as an effector, and the empty vector was used as an effector control in transient Luciferase assay performed in maize protoplasts. The vector with the Renilla reniformis (REN) gene driven by a $35 \mathrm{~S}$ promoter and firefly luciferase (LUC) gene driven by promoter sequence from $\mathrm{ZmBEH}$ were used as the reporter. The LUC/REN ratio represents the relative activity of the promoters. Error bars represent SD $(n=5)$ *represents $\mathrm{P}<0.05$ determined by Student's $\mathrm{t}$ test. $\mathrm{D}$, RNA-Seq data shows the expression profiles of $\mathrm{ZmBEH1}$ in different maturation stage of the ligular region (S0 to S4) in WT and $\lg 2$ mutant. Details showed in Supplemental Fig. S2, A and C. 
bioRxiv preprint doi: https://doi.org/10.1101/2021.02.02.429433; this version posted February 2, 2021. The copyright holder for this preprint (which was not certified by peer review) is the author/funder. All rights reserved. No reuse allowed without permission.

A

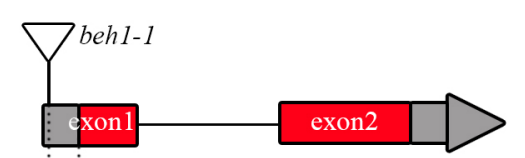

-288 ATG $(+1) \quad Z m B E H 1$

B

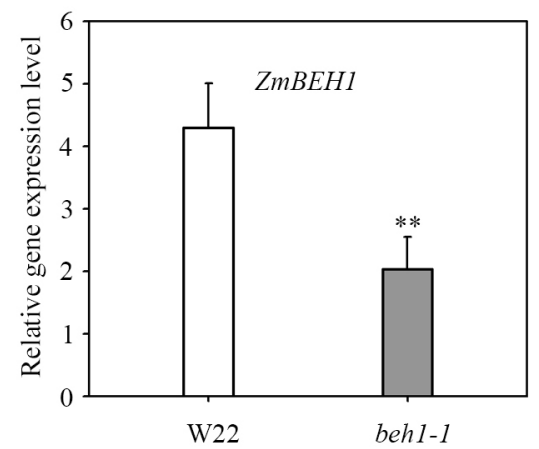

C
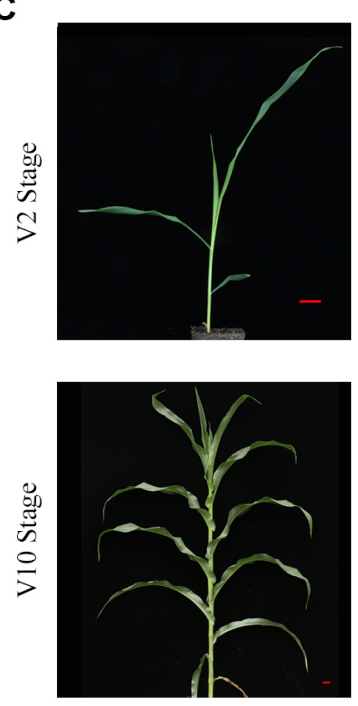

W22

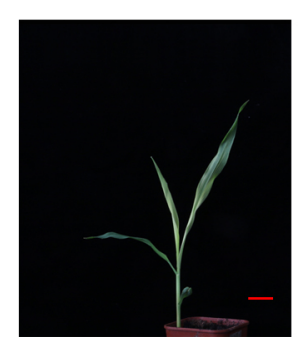

Bar, $3 \mathrm{~cm}$

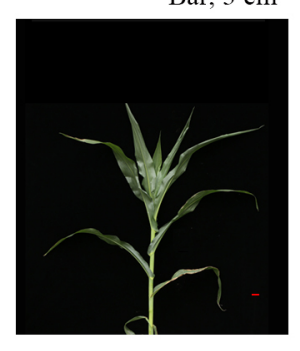

beh1-1 Bar, $5 \mathrm{~cm}$
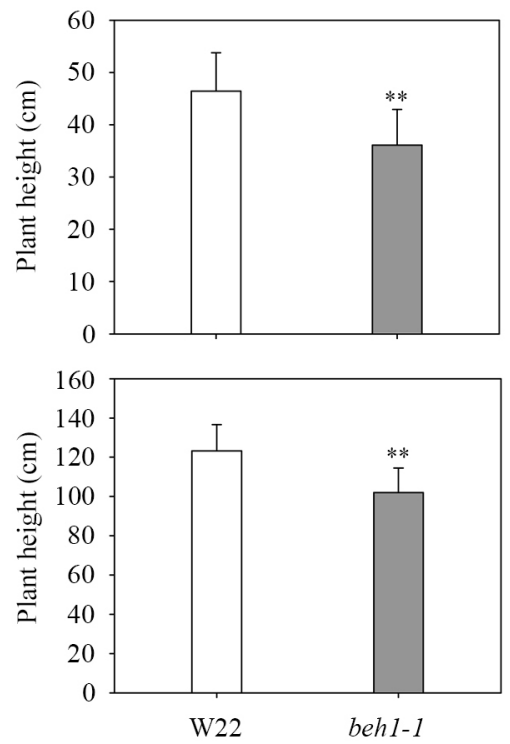

D

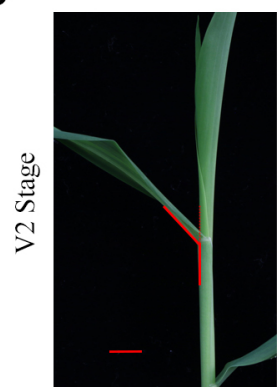

W22

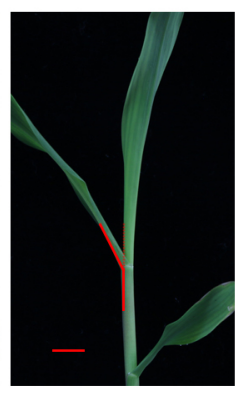

beh1-1 Bar, $1 \mathrm{~cm}$

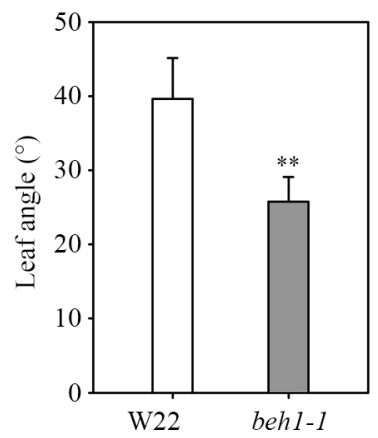

E

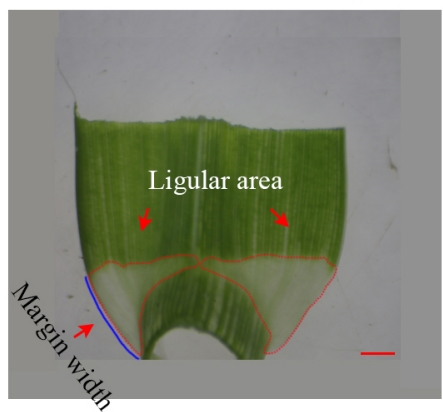

W22
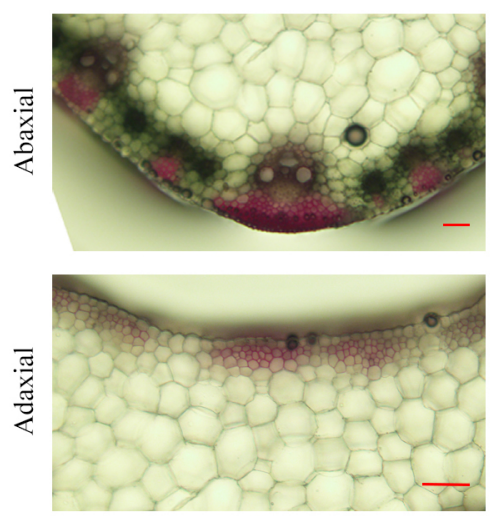

W22

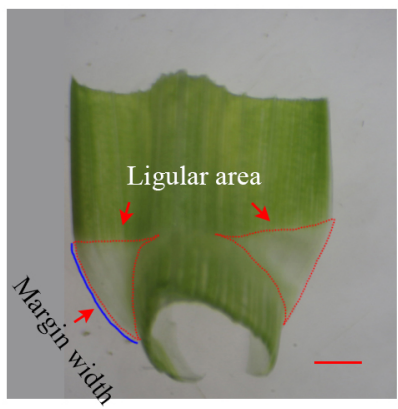

beh1-1 Bar, $1 \mathrm{~mm}$
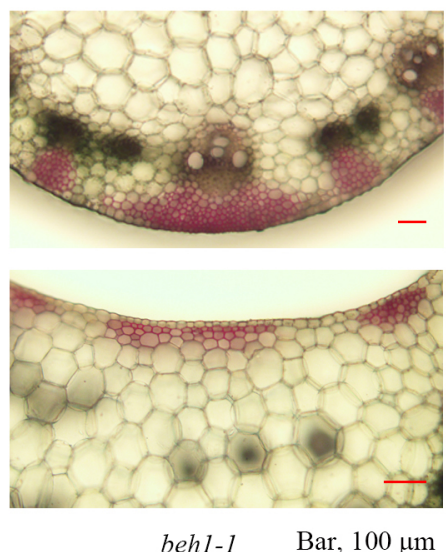

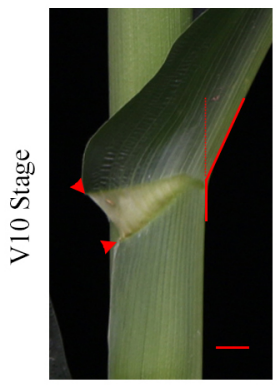

W22

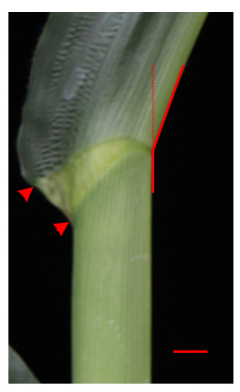

beh1-1 Bar, $1 \mathrm{~cm}$
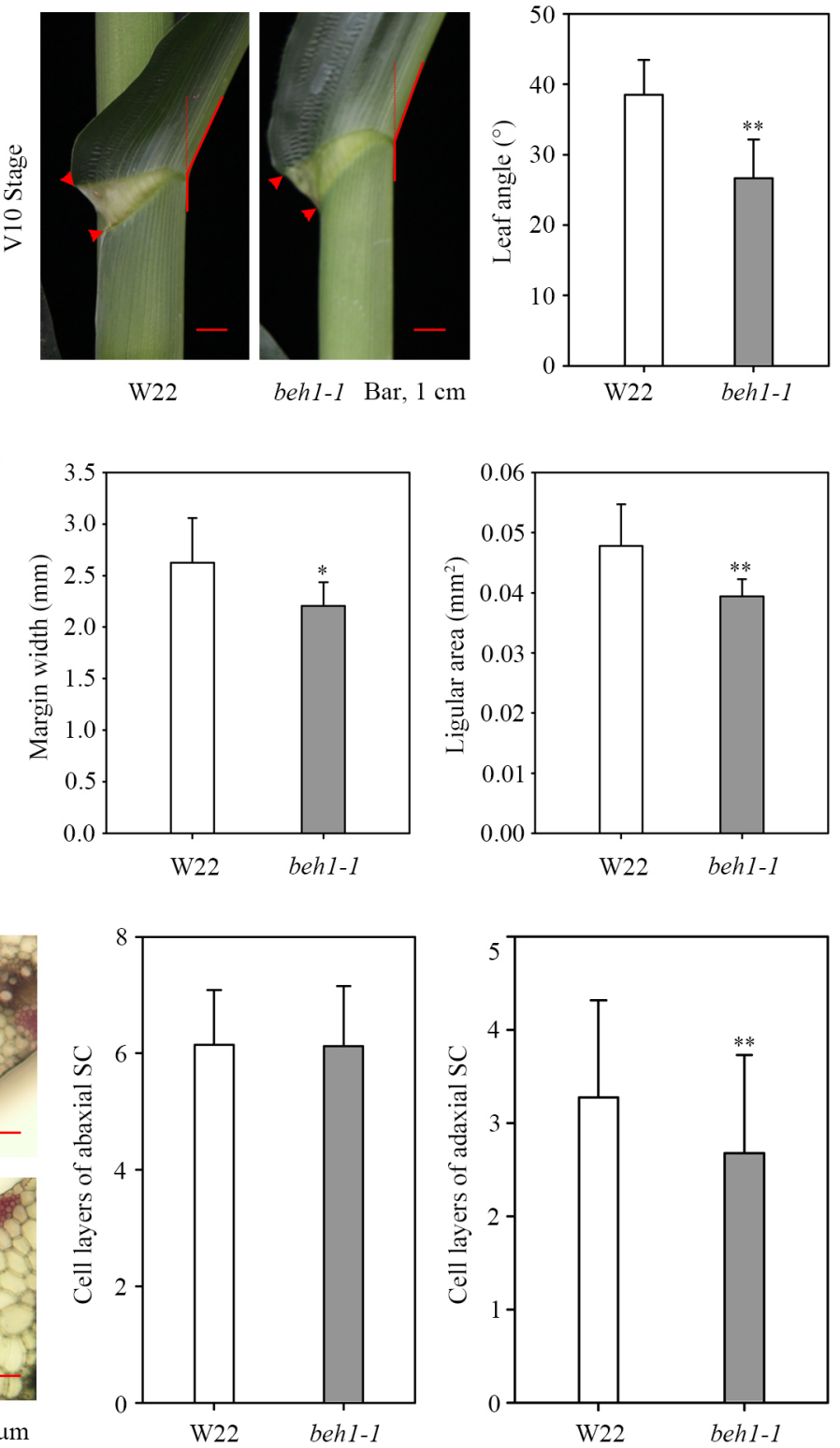

Figure 2. Phenotypes of beh1-1 plants. A, Uniform Mu-mediated insertion of ZmBEH1. A maize transposon-insertion line beh1-1 was obtained from the maize Uniform $\mathrm{Mu}$ resource, which carried a Mu insertion (UFMu-13557) in the 5'-UTR of ZmBEH1. B, Expression of ZmBEH1 gene in WT inbred line W22 and beh1-1 plants at V2 stage. **represents $\mathrm{P}<0.01$ determined by Student's t test. C, Plant height of WT inbred line W22 and behI-1 plants at V2 and V10 stages. From C to $\mathrm{E}$, * represents $\mathrm{P}<0.05$, **represents $\mathrm{P}<0.01$ determined by Student's t test, $\mathrm{n}=20$. D, Leaf angle changes between WT inbred line W22 and beh1-1 plants at V2 and V10 stages. E, Quantitative measurements of leaf ligule margin width and ligular area in WT inbred W22 and beh1-1 mutants at 15-day-old seedling stage. F, Cross-sections of the ligule from WT inbred line W22 and beh1-1 plants at V2 stage. The sclerenchyma cell (SC) layers stained red with safranin. Number of SC cell files at the adaxial and abaxial side were calculated from 10 replicates. Error bars are SD. $* * \mathrm{P}<0.01$. Scale bars, $100 \mu \mathrm{m}$. 
A

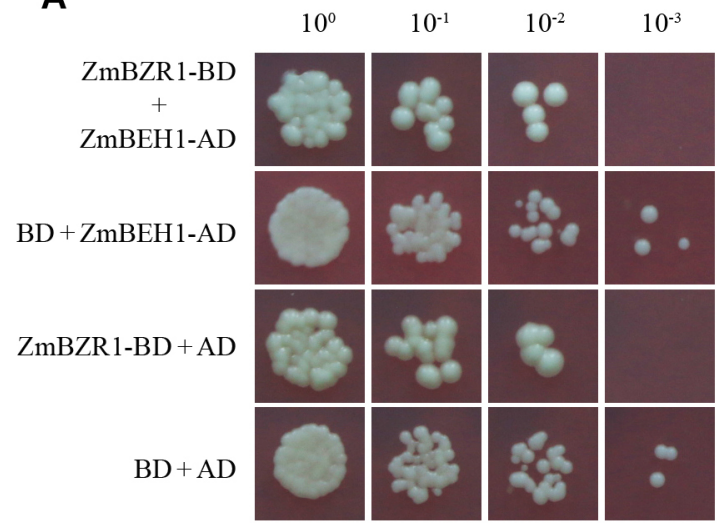

B

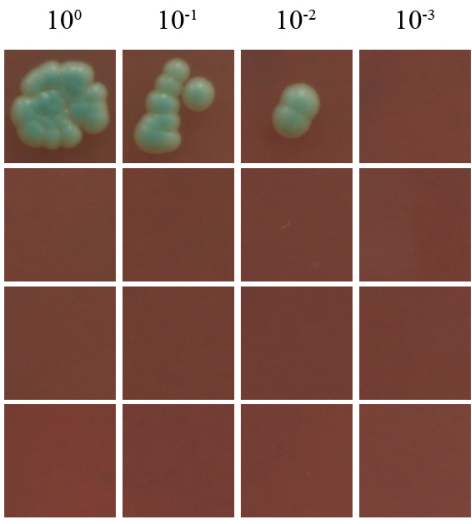

SD/-T-L-H-A+X- $\alpha-$ gal

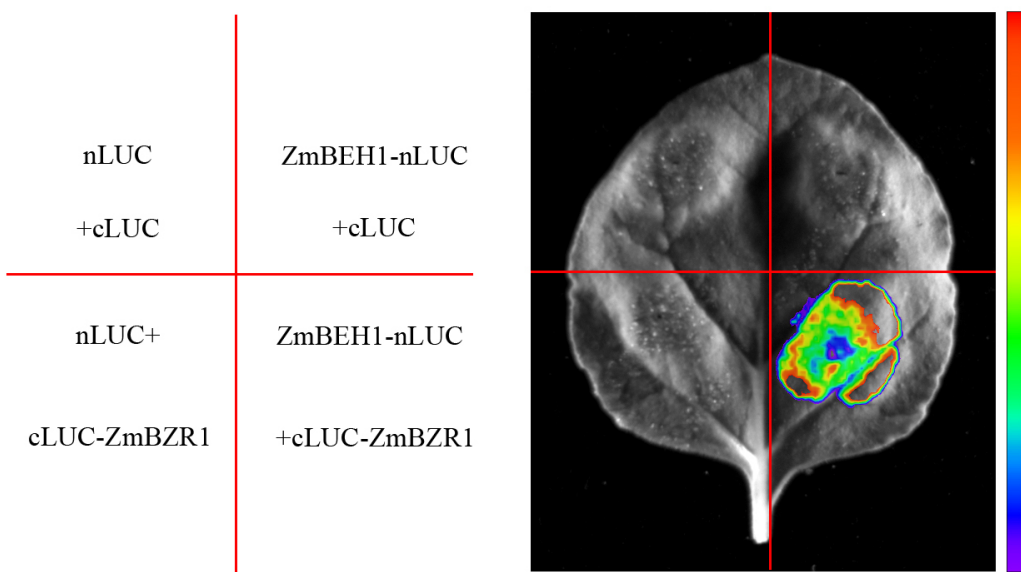

Figure 3. ZmBEH1 interacts with ZmBZR1. A, Interaction between ZmBZR1 and ZmBEH1 in yeast two-hybrid assays. Different concentrations of co-transformed yeast cells were spotted on synthetic dropout (SD) medium without tryptophan and leucine (SD/-T-L), or without tryptophan, leucine, histidine and adenine, and plus $20 \mathrm{mg} / \mathrm{mL} \mathrm{X- \alpha -gal} \mathrm{(SD/-T-L-H-A}+\mathrm{X}$ - $\alpha$-gal). B, Interaction between ZmBZR1 and $\mathrm{ZmBEH} 1$ in the split luciferase assay. 
A

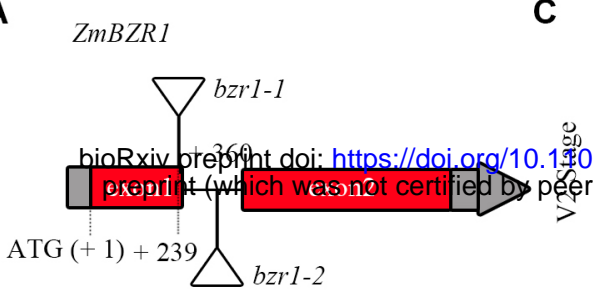

B
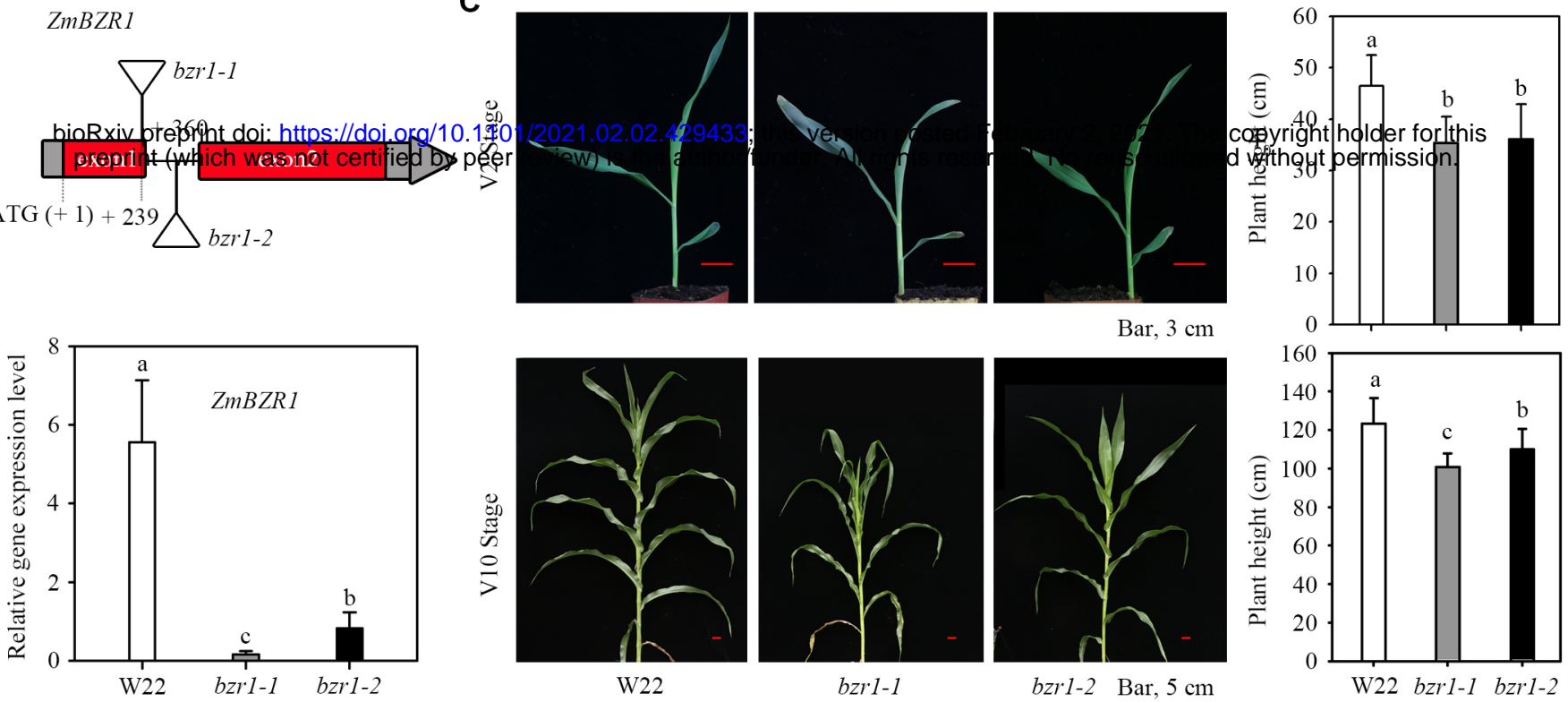

bzr1-1
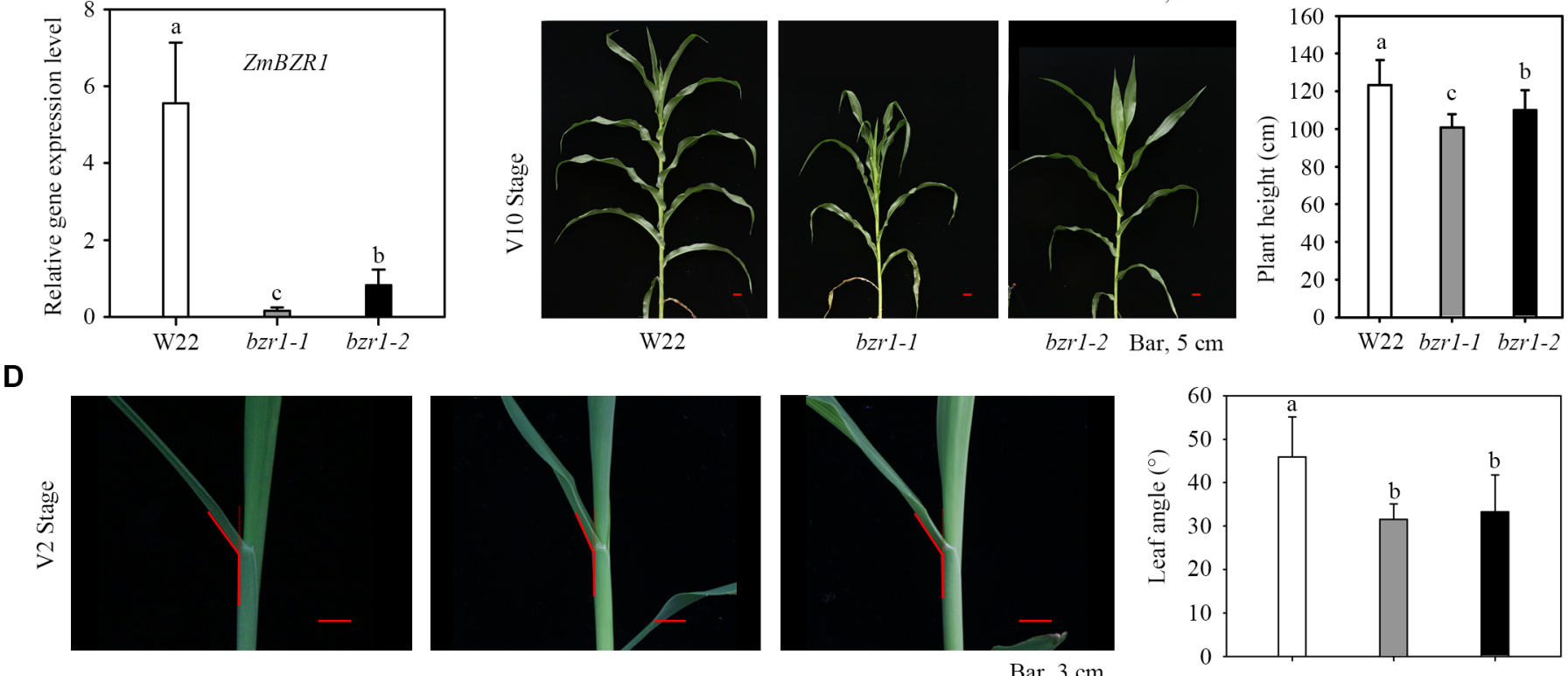

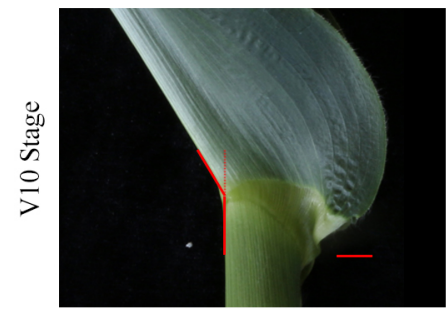

W22

E

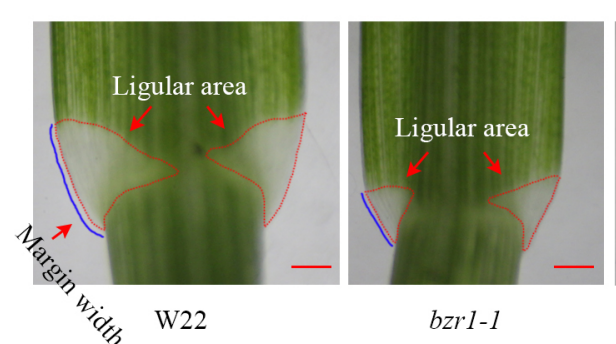

bzr $1-1$
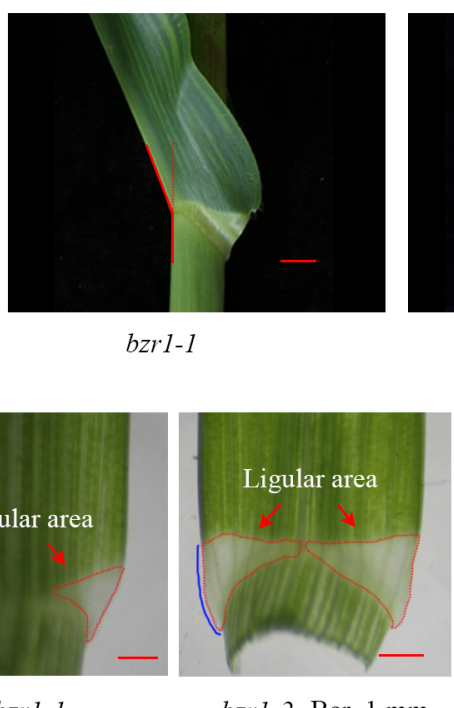

bzrl-2 Bar, $1 \mathrm{~mm}$

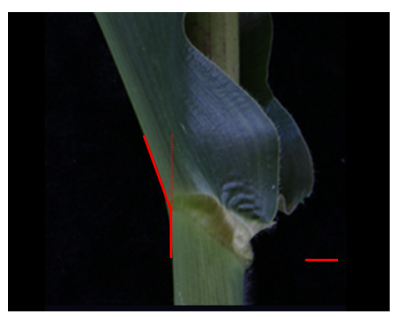

Bar, $1 \mathrm{~cm}$

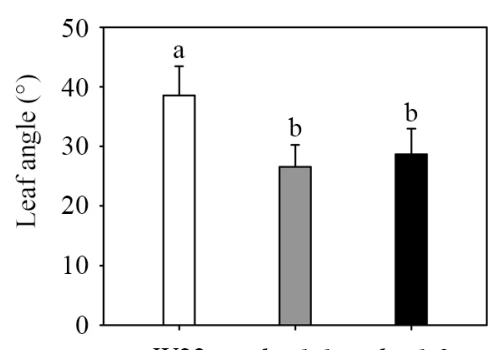

W22 bzrl-1 bzrl-2
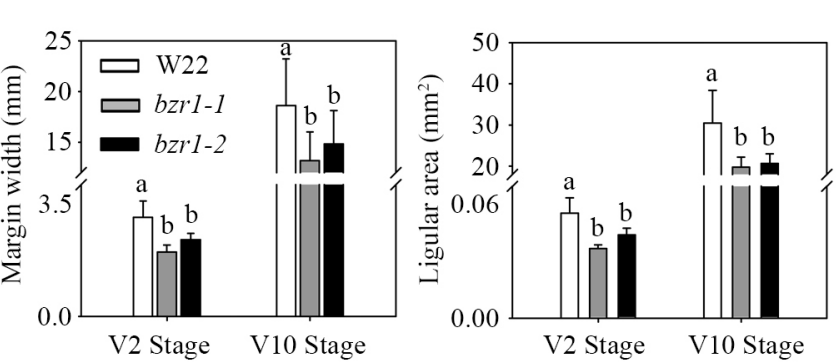

F
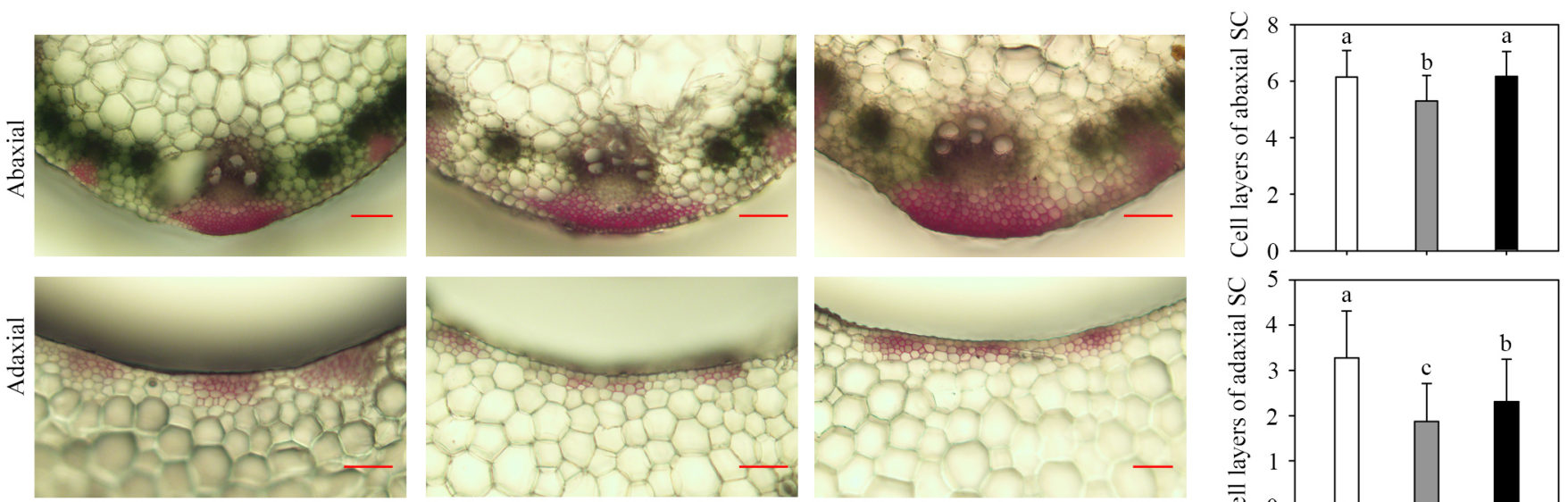

W22

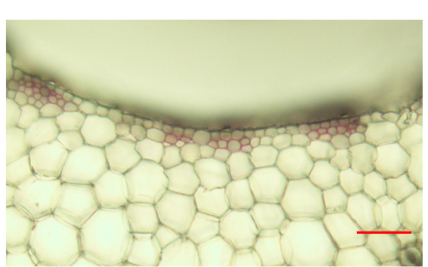

bzr1-1

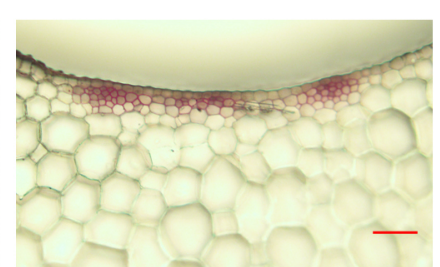

bzrl-2 Bar, $100 \mu \mathrm{m}$

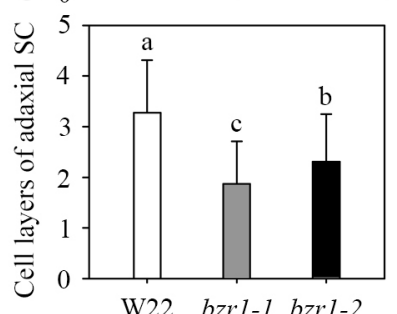

W22 bzrl-1 bzrl-2

Figure 4. Characterization of bzr1-1 and bzr1-2 mutants. A, Gene model shows the inserted position of Uniform Mu (UFMu-13537 and UFMu-03258) in the $1^{\text {st }}$ exon (bzr1-1) and $1^{\text {st }}$ intron (bzrl-2) of ZmBZR1 gene. B, Expression analysis of ZmBZR1 in WT inbred line W22 and bzr1-1, bzrl-2 plants at V2 stage. C, Plant height of WT inbred line W22 and bzrl-1, bzrl-2 plants in V2 and V10 stages. Different letters above the columns indicate statistically significant differences between groups $(\mathrm{n}=20)$. D, Leaf angle changes between WT inbred line W22 and bzrl-1, bzrl-2 plants in V2 and V10 stages ( $\mathrm{n}=20)$. E, Quantitative measurements of leaf ligule margin width and ligular area in WT inbred line W22 and bzrl-1, bzrl-2 plants at V2 and V10 stages (n=20). Different letters above the columns indicate statistically significant differences between groups. F, Cross-sections of the ligular region from WT inbred line W22 and bzr $1-1$, bzr $1-2$ plants at V2 stage. The sclerenchyma cell (SC) layers stained red with safranin. Number of SC cell files at the adaxial and abaxial side were calculated from 10 replicates. Error bars are SD. Scale bars, $100 \mu \mathrm{m}$. 
A

W22

bzr $1-1$

bzr1-2

beh1-1

bzr1-1 beh1-1

bzr1-2 beh1-1
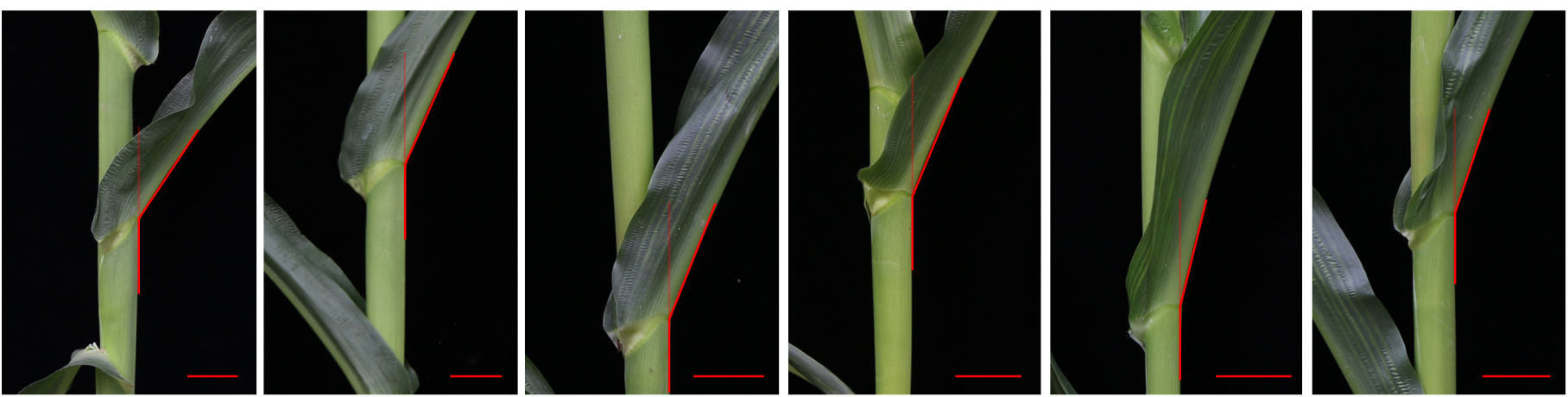

Bar, $3 \mathrm{~cm}$

B
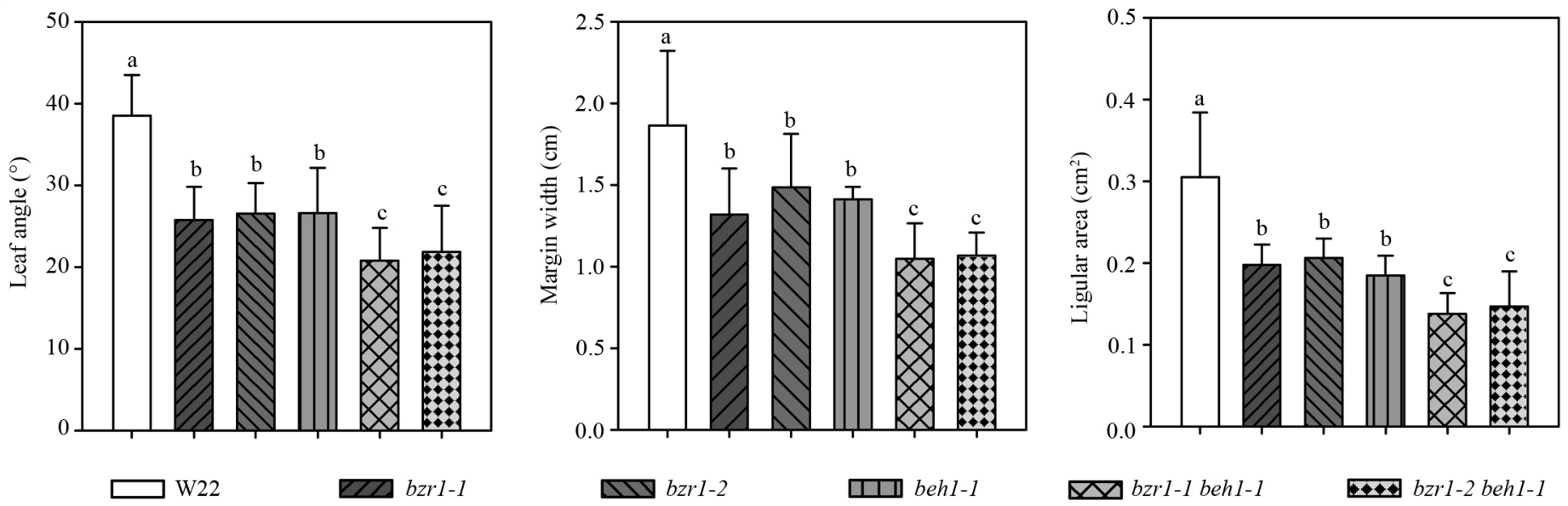

Figure 5. Leaf angle observation from single and double mutants of bzrl and beh1. A, Leaf angle morphology of WT inbred line W22, single mutants of bzrl-1, bzrl-2 and beh1-1, double mutants of bzrl-1 beh1-1 and bzrl-2 beh1-1. B, Quantitative measurements of leaf angle $(\mathrm{n}=20)$, margin width $(\mathrm{n}=10)$ and ligular area $(\mathrm{n}=10)$ in W22, single and double mutants. Different letters above the columns indicate statistically significant differences between groups. 
A

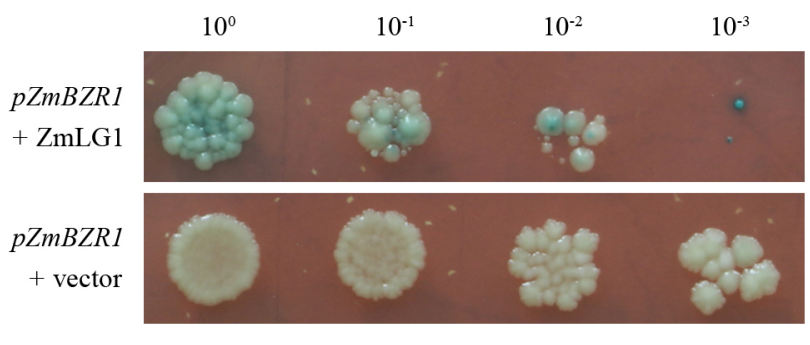

$\mathrm{SD} /$-Trp-Ura $+\mathrm{x}-\mathrm{gal}$
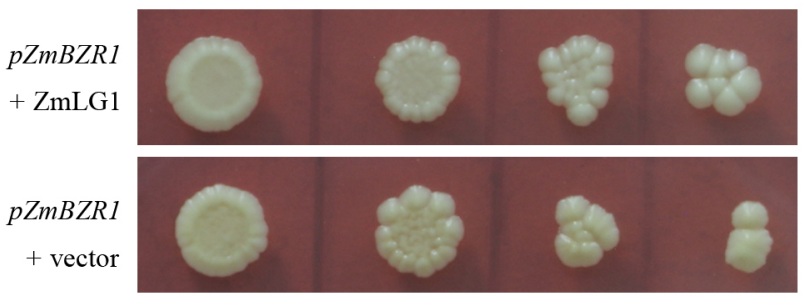

$\mathrm{SD} /$-Trp-Ura

C

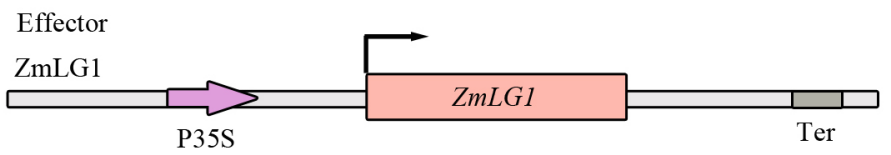

Control

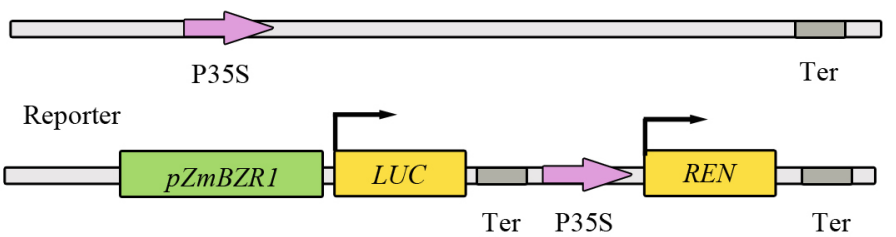

B

$\begin{array}{rccccc}\text { ZmLG1 } & - & - & + & + & + \\ \text { GST } & - & + & - & - & - \\ \text { Probe } & + & + & + & + & + \\ \text { competitor } & - & - & - & \times & \times \\ 8 & \ddot{~}\end{array}$

FP $\rightarrow$ atip

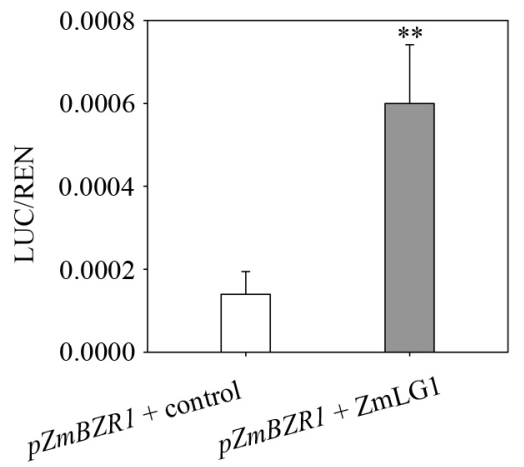

D

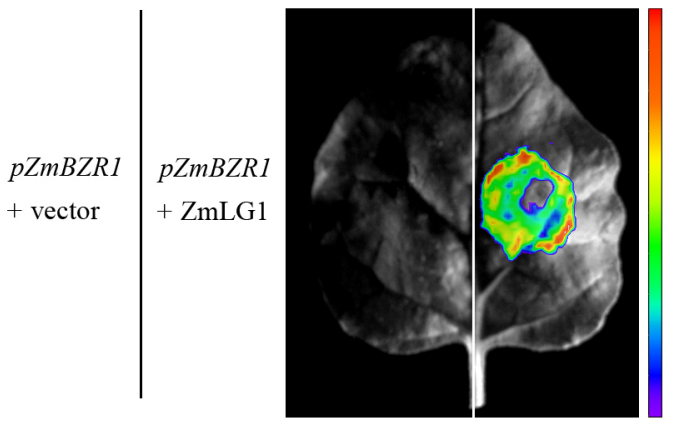

E

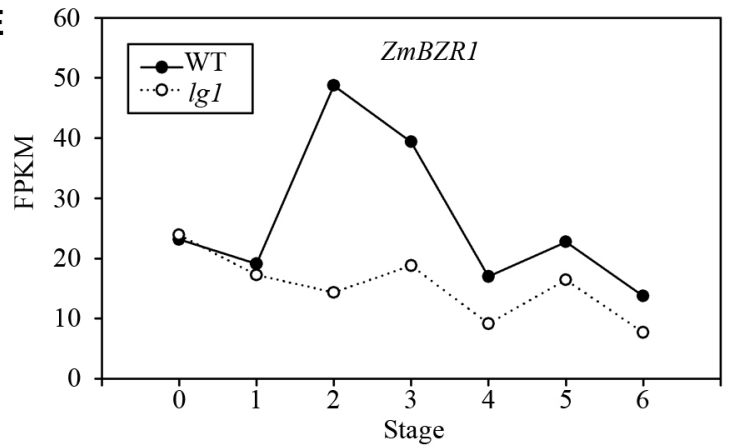

Figure 6. ZmLG1 directly activate the expression of ZmBZR1. A, ZmLG1 directly binds to the ZmBZR1 promoter in yeast one hybrid assay. B, ZmLG1 directly binds to the ZmBZRI promoter in EMSA. "-" indicates the absence of proteins. "+" indicate the present; FP indicates free probe. $\mathrm{C}, \mathrm{ZmLG} 1$ actives the promoter of $Z m B Z R 1$ in Dual-luciferase assay. The coding sequence of LG1 driven by the $35 \mathrm{~S}$ promoter was used as an effector, and the empty vector was used as an effector control in transient luciferase assay performed in maize protoplasts. The vector with the Renilla reniformis (REN) gene driven by a $35 \mathrm{~S}$ promoter and firefly luciferase (LUC) gene driven by promoter sequence from $Z m B Z R I$ were used as the reporter. The LUC/REN ratio represents the relative activity of the promoters. Error bars represent SD $(\mathrm{n}=5) * *$ represents $\mathrm{P}<0.01$ determined by Student's $\mathrm{t}$ test. D, Transient luciferase intensity assay performed in tobacco leaves showing that ZmLG1 actives the promoter of ZmBZR1. E, RNA-Seq data shows the expression of ZmBZR1 in different mature stage of ligular region (S0 to S4) in WT and $\lg I$ mutant. Details showed in Supplemental Fig. S2. 
A

$\begin{array}{llllllll}p Z m S C L 28 & p Z m S C L 28 & p Z m S C L 28 & p Z m S C L 28 & p Z m S C L 28 & p Z m S C L 28 & p Z m S C L 28 & p Z m S C L 28 \\ + \text { ZmBZR1 } & + \text { ZmBEH1 } & + \text { ZmBZR1 } & + \text { Vector } & + \text { ZmBZR1 } & + \text { ZmBEH1 } & + \text { ZmBZR1 } & + \text { Vector } \\ & & + \text { ZmBEH1 } & & & & + \text { ZmBEH1 }\end{array}$

bioRxiv preprint doi: https://doi.org/10.1101/2021.02.02.429433; this version posted February 2, 2021. The copyright holder for this preprint (which was not certified by peer review) is the author/funder. All rights reserved. No reuse allowed without permission. preprint (which was not certiled by peer review) is the author: $\rightarrow$

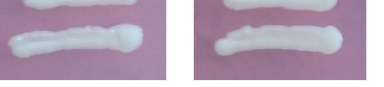

SD/-Trp-Ura

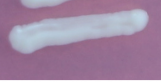

$Y^{s c l 28-1}$

B

ZmSCL28

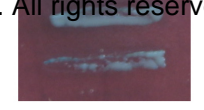

20 $\longrightarrow$

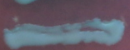

$x=\infty$

\section{5'-UTR}

SD/-Trp-Ura + x-gal

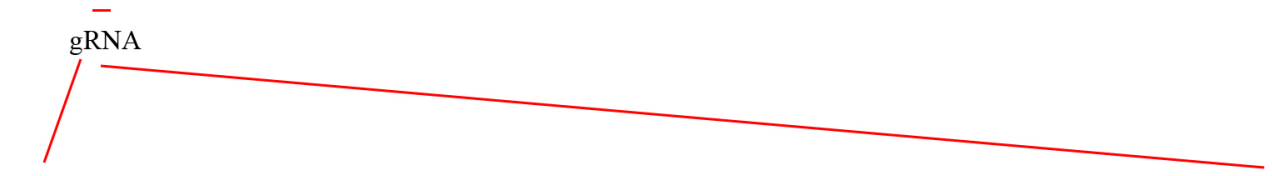

KN5585 TTCTCCAAGCGGGCGAGCAACCG CGGCGATGGCTCCGGCGCCGCGCCGCGCGTCGCGGCGCCCGA

scl28-2 TTCTCCAAGCG -

C

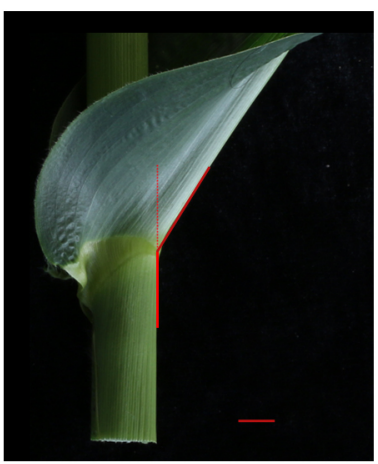

W22

D

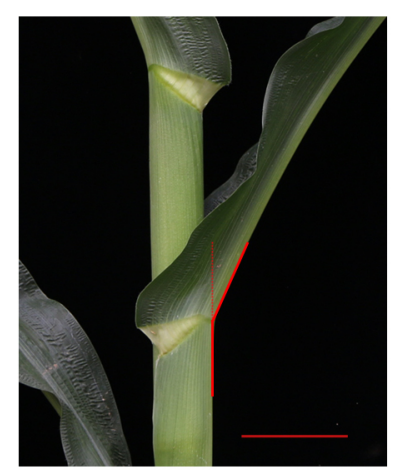

W22

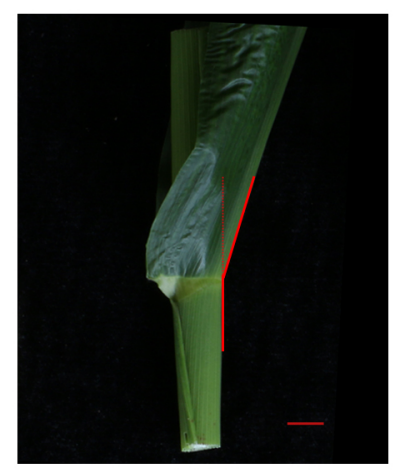

scl28-1

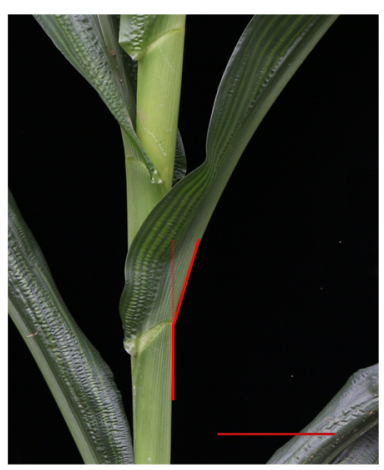

bzr1-1 scl28-1

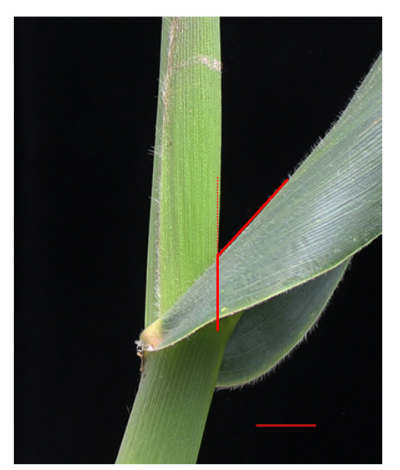

KN5585

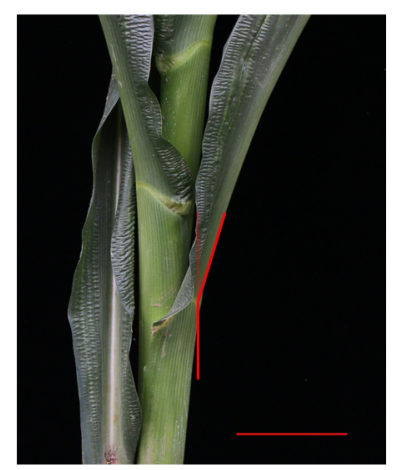

bzr1-2 scl28-1

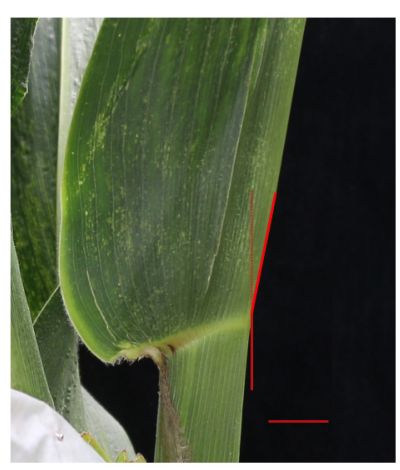

scl28-2 Bar, $5 \mathrm{~cm}$

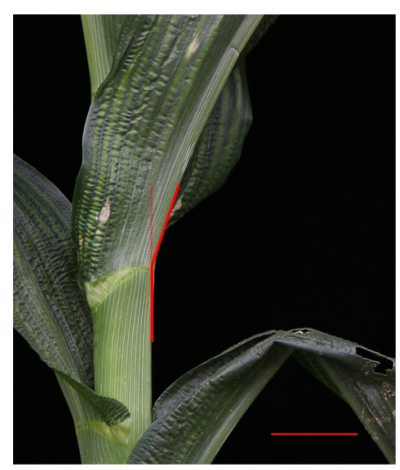

beh1-1 scl28-1 Bar, $5 \mathrm{~cm}$

E
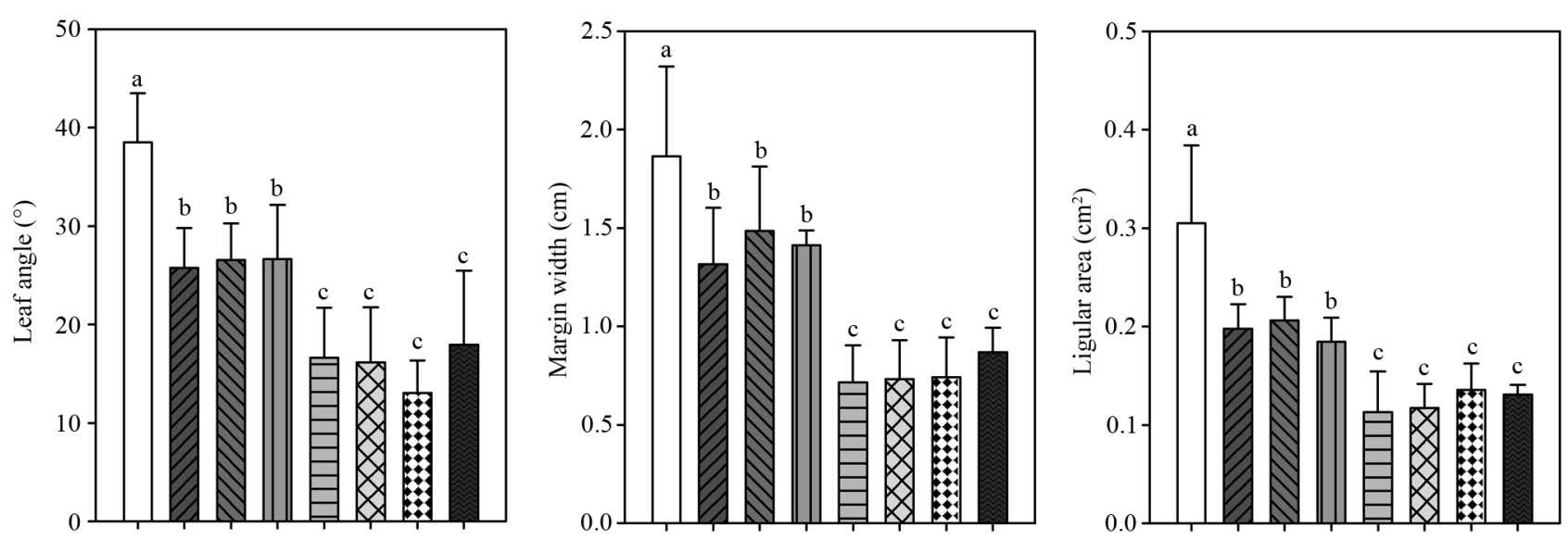

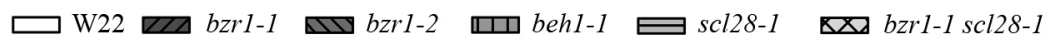

bzrl-2 scl28-1

beh1-1 scl28-1

Figure7. ZmSCL28 works downstream of ZmBZR1 and ZmBEH1. A, Y1H assays showed that ZmBZR1 and ZmBEH1 bound to the promoter of ZmSCL28. B, Gene model presents the positions of two mutant alleles in ZmSCL28. The triangle shows the position of a Mu insertion (UFMu-09491) from line $s c l 28-1$ at W22 genetic background, and red line shows the position of a single-guide RNA (sgRNA) designed to mutate the ZmSCL28 using the CRISPR/Cas9 technology. The sequence of sgRNA is highlighted in blue, and the symbols "..." indicate the deletion caused by CRISPR/Cas9-induced mutations in line scl28-2 at KN5585 genetic background. C, Leaf angle morphology in the WT inbred line W22 and mutant scl28-1, WT inbred line KN5585 and mutant $s c l 28-2$ at V10 stage. Scale bars $=5 \mathrm{~cm}$. D, Leaf angle morphology in the WT W22 and double mutants of bzr1-1 scl28-1, bzrl-2 scl28-1 and beh1-1 scl28-1 at V10 stage. Scale bars $=5 \mathrm{~cm}$. E, Quantitative measurements of leaf angle $(\mathrm{n}=20)$, margin width $(\mathrm{n}=10)$ and ligular area $(n=10)$ in single and double mutants. Different letters above the columns indicate statistically significant differences between groups. 


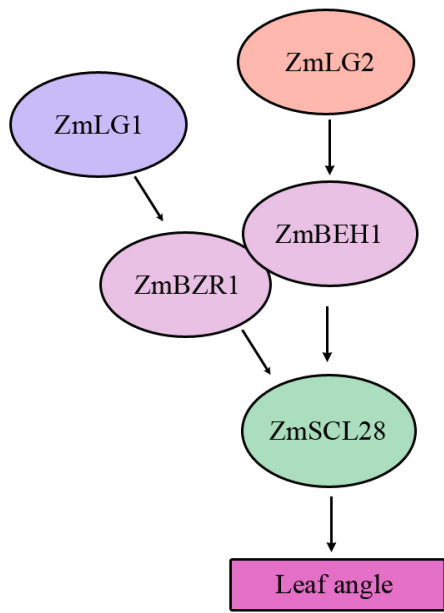

(cell wall, cell elongation...)

Figure 8. A proposed ZmLG2 (LG1)-ZmBEH1 (BZR1)-ZmSCL28 module regulates leaf angle in maize. The $\mathrm{ZmLG} 2$ binds to the protomer of $\mathrm{ZmBEH} 1$ to activates its expression. The $\mathrm{ZmBEH} 1$ interact with $\mathrm{ZmBZR} 1$, which is a direct target of ZmLG1, to regulate the expression of a downstream target gene, $\mathrm{ZmSCL28}$. These proposed regulatory cascades may control the leaf angle size in maize. 


\section{Parsed Citations}

Bai MY, Zhang LY, Gampala SS, Zhu SW, Song WY, Chong K, Wang ZY (2007) Functions of OsBZR1 and 14-3-3 proteins in brassinosteroid signaling in rice. Proc Natl Acad Sci USA 104: 13839-13844

Google Scholar: Author Only Title Only Author and Title

Bolduc N, O'Connor D, Moon J, Lewis M, Hake S (2012) How to pattern a leaf. Cold Spring Harb Symp Quant Biol 77: 47-51

Google Scholar: Author Only Title Only Author and Title

Cao Y, Zeng H, Ku L, Ren Z, Han Y, Su H, Dou D, Liu H, Dong Y, Zhu F, Li T, Zhao Q, Chen Y (2020) ZmIBH1-1 regulates plant architecture in maize. J Exp Bot 71: 2943-2955

Google Scholar: Author Only Title Only Author and Title

Chen LG, Gao Z, Zhao Z, Liu X, Li Y, Zhang Y, Liu X, Sun Y, Tang W (2019) BZR1 family transcription factors function redundantly and indispensably in BR signaling but exhibit BRI1-independent function in regulating anther development in Arabidopsis. Mol Plant 12: 1408-1415

Google Scholar: Author Only Title Only Author and Title

Duvick DN (2005) Genetic progress in yield of United States maize (Zea mays L.). Maydica: 193-202

Google Scholar: Author Only Title Only Author and Title

Muehlbauer GJ, Fowler JE, Girard L, Tyers R, Harper L, Freeling M (1999) Ectopic expression of the maize homeobox gene liguleless3 alters cell fates in the leaf. Plant Physiol 119: 651-662

Google Scholar: Author Only Title Only Author and Title

Harper L, Freeling M (1996) Interactions of liguleless1 and liguleless2 function during ligule induction in maize. Genetics 144: 1871 1882

Google Scholar: Author Only Title Only Author and Title

Hirano K, Yoshida H, Aya K, Kawamura M, Hayashi M, Hobo T, Sato-Izawa K, Kitano H, Ueguchi-Tanaka M, Matsuoka M (2017) Small organ size 1 and small organ size 2/dwarf and low tillering form a complex to integrate auxin and brassinosteroid signaling in rice. Mol Plant 10: 590-604

Google Scholar: Author Only Title Only Author and Title

Kir G, Ye H, Nelissen H, Neelakandan AK, Kusnandar AS, Luo A, Inzé D, Sylvester AW, Yin Y, Becraft PW(2015) RNA interference knockdown of BRASSINOSTEROID INSENSITIVE1 in maize reveals novel functions for brassinosteroid signaling in controlling plant architecture. Plant Physiol 169: 826-839

Google Scholar: Author Only Title Only Author and Title

Ku L, Wei X, Zhang S, Zhang J, Guo S, Chen Y (2011) Cloning and characterization of a putative TAC1 ortholog associated with leaf angle in maize (Zea mays L.). PLoS ONE 6: e20621

Google Scholar: Author Only Title Only Author and Title

Lambert RJ, Johnson RR (1978) Leaf angle, tassel morphology, and the performance of maize hybrids. Crop Sci 18: $499-502$ Google Scholar: Author Only Title Only Author and Title

Li H, Ye K, Shi Y, Cheng J, Zhang X, Yang S (2017) BZR1 Positively Regulates Freezing Tolerance via CBF-Dependent and CBFIndependent Pathways in Arabidopsis. Mol Plant 10: 545-559

Google Scholar: Author Only Title Only Author and Title

Li JM, Jin H (2007) Regulation of brassinosteroid signaling. Trends Plant Sci 12: 37-41

Google Scholar: Author Only Title Only Author and Title

Luo XM, Lin WH, Zhu S, Zhu JY, Sun Y, Fan XY, Cheng M, Hao Y, Oh E, Tian M, Liu L, Zhang M, Xie Q, Chong K, Wang ZY (2010)

Integration of light- and brassinosteroid-signaling pathways by a GATA transcription factor in Arabidopsis. Dev Cell 19: 872-883

Google Scholar: Author Only Title Only Author and Title

Makarevitch I, Thompson A, Muehlbauer GJ, Springer NM (2012) Brd1 gene in maize encodes a brassinosteroid C-6 oxidase. PLoS ONE 7: e30798.

Google Scholar: Author Only Title Only Author and Title

Mantilla-Perez MB, Salas Fernandez MG (2017) Differential manipulation of leaf angle throughout the canopy: current status and prospects. J Exp Bot 68: 5699-5717

Google Scholar: Author Only Title Only Author and Title

Moreno MA, Harper LC, Krueger RW, Dellaporta SL, Freeling Michael (1997) liguleless1 encodes a nuclear-localized protein required for induction of ligules and auricles during maize leaf organogenesis. Genes Dev: 616-628

Google Scholar: Author Only Title Only Author and Title

Min HJ, Cui LH, Oh TR, Kim JH, Kim TW, Kim WT (2019) OsBZR1 turnover mediated by OsSK22-regulated U-box E3 ligase OsPUB24 in rice BR response. Plant J 99: 426-438

Google Scholar: Author Only Title Only Author and Title 
Moreno MA, Harper LC, Krueger RW, Dellaporta SL, Freeling M (1997) liguleless1 encodes a nuclear-localized protein required for induction of ligules and auricles during maize leaf organogenesis. Genes Dev 11: 616-628

Google Scholar: Author Only Title Only Author and Title

Pendleton JW, Smith GE, Winter SR, Johnston TJ (1968) Field investigations of the relationships of leaf angle in corn (Zea mays L.) to grain yield and apparent photosynthesis. Agronomy Journal 60: 422-424

Google Scholar: Author Only Title Only Author and Title

Qiao S, Sun S, Wang L, et al. (2017) The RLA1/SMOS1 transcription factor functions with OsBZR1 to regulate brassinosteroid signaling and rice architecture. Plant Cell 29: 292-309

Google Scholar: Author Only Title Only Author and Title

Ren Z, Wu L, Ku L, Wang H, Zeng H, Su H, Wei L, Dou D, Liu H, Cao Y, Zhang D, Han S, Chen Y (2020) ZmILI1 regulates leaf angle by directly affecting liguleless 1 expression in maize. Plant Biotechnol J 18: 881-883

Google Scholar: Author Only Title Only Author and Title

Saito M, Kondo Y, Fukuda H (2018) BES1 and BZR1 redundantly promote phloem and xylem differentiation. Plant Cell Physiol 59: 590600

Google Scholar: Author Only Title Only Author and Title

Song Z, Yan T, Liu J, Bian Y, Heng Y, Lin F, Jiang Y, Wang Deng X, Xu D (2020) BBX28/BBX29, HY5 and BBX30/31 form a feedback loop to fine-tune photomorphogenic development. Plant J 104: 377-390

Google Scholar: Author Only Title Only Author and Title

Sun S, Chen D, Li X, Qiao S, Shi C, Li C, Shen H, Wang X (2015) Brassinosteroid signaling regulates leaf erectness in Oryza sativa via the control of a specific U-type cyclin and cell proliferation. Dev Cell 34: 220-228

Google Scholar: Author Only Title Only Author and Title

Tian J, Wang C, Xia J, Wu L, Xu G, Wu W, Li D, Qin W, Han X, Chen Q, Jin W, Tian F (2019) Teosinte ligule allele narrows plant architecture and enhances high-density maize yields. Science 365: 658-664

Google Scholar: Author Only Title Only Author and Title

Tong H, Chu C (2009) Roles of DLT in fine modulation on brassinosteroid response in rice. Plant Signal Behav 4: $438-439$

Google Scholar: Author Only Title Only Author and Title

Tong H, Jin Y, Liu W, Li F, Fang J, Yin Y, Qian Q, Zhu L, Chu C (2009) DWARF AND LOW-TILLERING, a new member of the GRAS family, plays positive roles in brassinosteroid signaling in rice. Plant $\mathrm{J}$ 58: 803-816

Google Scholar: Author Only Title Only Author and Title

Tong H, Liu L, Jin Y, Du L, Yin Y, Qian Q, Zhu L, Chu C (2012) DWARF AND LOW-TILLERING acts as a direct downstream target of a GSK3/SHAGGY-like kinase to mediate brassinosteroid responses in rice. Plant Cell 24: 2562-2577

Google Scholar: Author Only Title Only Author and Title

Tu X, Mejía-Guerra MK, Valdes Franco JA, Tzeng D, Chu PY, Shen W, Wei Y, Dai X, Li P, Buckler ES, Zhong S (2020) Reconstructing the maize leaf regulatory network using ChIP-seq data of 104 transcription factors. Nat Commun 11: 5089

Google Scholar: Author Only Title Only Author and Title

Walsh J, Waters CA, Freeling M (1998) The maize gene liguleless2 encodesa basic leucine zipper protein involvedin the establishment of the leaf blade-sheath boundary. Genes Dev 12: 208-218

Google Scholar: Author Only Title Only Author and Title

Wang W, Bai MY, Wang ZY (2014) The brassinosteroid signaling network-a paradigm of signal integration. Curr Opin Plant Biol 21: 147153

Google Scholar: Author Only Title Only Author and Title

Wang ZY, Bai MY, Oh E, Zhu JY (2012) Brassinosteroid signaling network and regulation of photomorphogenesis. Annu Rev Genet 46: 701-724

Google Scholar: Author Only Title Only Author and Title

Wei H, Zhao Y, Xie Y, Wang H (2018) Exploiting SPL genes to improve maize plant architecture tailored for high density planting. J Exp Bot 20:4675-4688

Google Scholar: Author Only Title Only Author and Title

Xing HL, Dong L, Wang ZP, Zhang HY, Han CY, Liu B, Wang XC, Chen QJ (2014) ACRISPR/Cas9 toolkit for multiplex genome editing in plants. BMC Plant Biol 14: 327

Google Scholar: Author Only Title Only Author and Title

Zhang J, Ku LX, Han ZP, Guo SL, Liu HJ, Zhang ZZ, Cao LR, Cui XJ, Chen YH (2014) The ZmCLA4 gene in the qLA4-1 QTL controls leaf angle in maize (Zea mays L.). J Exp Bot 65: 5063-5076

Google Scholar: Author Only Title Only Author and Title

Zhao Y, Wang H, Bo C, Dai W, Zhang X, Cai R, Gu L, Ma Q, Jiang H, Zhu J, Cheng B (2018) Genome-wide association study of maize plant architecture using $F 1$ populations. Plant Mol Biol 99:1-15

Google Scholar: Author Only Title Only Author and Title 
$\longrightarrow$ 